\title{
Probing the formation of intermediate- to high-mass stars in protoclusters
}

\section{A detailed millimeter study of the NGC 2264 clumps}

\author{
N. Peretto ${ }^{1}$, Ph. André1,2, and A. Belloche ${ }^{3}$ \\ ${ }^{1}$ CEA/DSM/DAPNIA, Service d'Astrophysique, CE Saclay, Orme des Merisiers, 91191 Gif-sur-Yvette, France \\ e-mail: [peretto; pandre]@cea.fr \\ 2 AIM - Unité Mixte de Recherche CEA - CNRS - Université Paris VII - UMR 7158, France \\ 3 Max Planck Institut für Radioastronomie, Auf dem Hügel 69, 53121 Bonn, Germany
}

Received 27 April 2005 / Accepted 24 August 2005

ABSTRACT

We present the results of dust continuum and molecular line observations of two massive cluster-forming clumps, NGC 2264-C and NGC 2264-D, including extensive mapping performed with the MAMBO bolometer array and the HERA heterodyne array on the IRAM $30 \mathrm{~m}$ telescope. Both NGC 2264 clumps are located in the Mon OB1 giant molecular cloud complex, adjacent to one another. Twelve and fifteen compact millimeter continuum sources (i.e. MMSs) were identified in clumps C and D, respectively. These MMSs have larger sizes and masses than the millimeter continuum condensations detected in well-known nearby protoclusters such as $\rho$ Ophiuchi. The MMSs of NGC 2264 are closer in size to the $\mathrm{DCO}^{+}$"cores" of $\rho$ Oph, although they are somewhat denser and exhibit broader linewidths. Most of the MMSs of NGC 2264-C harbor candidate Class 0 protostars associated with shocked molecular hydrogen jets. Evidence of widespread infall motions was found in, e.g., $\mathrm{HCO}^{+}(3-2)$ or $\mathrm{CS}(3-2)$ in both NGC 2264-C and NGC 2264-D. A sharp velocity discontinuity $\sim 2 \mathrm{~km} \mathrm{~s}^{-1}$ in amplitude was observed in $\mathrm{N}_{2} \mathrm{H}^{+}(1-0)$ and $\mathrm{H}^{13} \mathrm{CO}^{+}(1-0)$ in the central, innermost part of NGC 2264-C, which we interpreted as the signature of a strong dynamical interaction between two MMSs and their possible merging with the central MMS C-MM3. Radiative transfer modelling supports the idea that NGC 2264-C is a highly unstable prolate clump in the process of collapsing along its long axis on a near free-fall dynamical timescale $\sim 1.7 \times 10^{5} \mathrm{yr}$. Our model fit of this large-scale collapse suggests a maximum mass inflow rate $\sim 3 \times 10^{-3} M_{\odot} \mathrm{yr}^{-1}$ toward the central protostellar object C-MM3. In NGC 2264-D, we estimated a mass infall rate $\dot{M}_{\mathrm{DMM} 1} \sim 1.1 \times 10^{-4} M_{\odot} \mathrm{yr}^{-1}$ toward the rotating Class 0 object D-MM1, also based on radiative transfer modelling of the observations. Such infall rates are sufficiently high to overcome radiation pressure and allow the formation of $\sim 20 M_{\odot}$ stars by accretion in $\sim 1.7 \times 10^{5} \mathrm{yr}$, i.e., a time that is similar to the global dynamical timescale of the central part of NGC 2264-C. We conclude that we are likely witnessing the formation of a high-mass $\left(\gtrsim 10 M_{\odot}\right)$ protostar in the central part of NGC 2264-C. Our results suggest a picture of massive star formation intermediate between the scenario of stellar mergers of Bonnell et al. (1998) and the massive turbulent core model of McKee \& Tan (2003), whereby a turbulent, massive ultra-dense core is formed by the gravitational merger of two or more Class 0 protostellar cores at the center of a collapsing protocluster.

Key words. stars: formation - stars: circumstellar matter - ISM: clouds - ISM: molecules - ISM: kinematics and dynamics

\section{Introduction}

While most stars are believed to form in clusters (e.g. Adams \& Myers 2001; Lada \& Lada 2003), our present theoretical understanding of the star formation process is essentially limited to isolated dense cores and protostars (e.g. Shu et al. 1987, 2004). Detailed studies of the earliest phases of clustered star formation are crucially needed if we are to explain the origin of the stellar initial mass function (IMF) and the birth of massive stars. There is indeed a growing body of evidence that highmass $\left(M_{\star}>8 M_{\odot}\right)$ stars may be able to form only in a clustered environment (e.g. Zinnecker et al. 1993; Testi et al. 1999; Clarke et al. 2000).
On the theoretical side, two main scenarios have been proposed for clustered star formation in turbulent molecular clouds. In the first scenario, the distribution of stellar masses is primarily determined by turbulent fragmentation. Briefly, self-gravitating condensations (each containing one local Jeans mass) form as turbulence-generated density fluctuations (e.g. Padoan \& Nordlund 2002), then decouple from their turbulent environment (e.g. Myers 1998), and eventually collapse with little interaction with their surroundings. In this scenario, high-mass stars are built-up by a scaled-up version of the accretion process believed to be at work in low-mass protostars: a high accretion rate $\left(>10^{-3} M_{\odot} \mathrm{yr}^{-1}\right)$, generated by the high pressure of the turbulent environment (e.g. McKee \& Tan 2003) 
and/or the influence of an external trigger (e.g. Hennebelle et al. 2003), is sufficient to overcome the radiation pressure that would normally halt the collapse soon after $M_{\star} \approx 8 M_{\odot}$ (e.g. Stahler et al. 2000).

By contrast, in the second scenario, the distribution of stellar masses results solely from the dynamics of the parent protocluster. Here, individual protostellar seeds have large proper motions with respect to the mean cloud velocity. They accrete cloud material competitively while orbiting in the gravitational potential well of a collapsing protocluster and possibly collide with one another (e.g. Bonnell et al. 1998, 2001). In this alternative scenario, competitive accretion and dynamical interactions between individual cluster members play a dominant role (e.g. Klessen \& Burkert 2000; Bate et al. 2003). In the dense central region of the collapsing protocluster, the probability of encounters becomes high enough that massive stars can form by the coalescence of intermediate-mass protostars (e.g. Bonnell \& Bate 2002). Determining which of these two pictures dominates in actual cluster-forming clouds is a major issue that is still open.

As one expects the internal dynamics of protoclusters to differ markedly in the two pictures, comprehensive molecular line studies of the kinematics of prestellar condensations and Class 0 protostars in cluster-forming regions can provide powerful observational tests (cf. André 2002). In an effort to improve our knowledge of the dynamics of protoclusters and to constrain theoretical models of clustered star formation, we carried out a combination of millimeter dust continuum and molecular line observations of the active cluster-forming region NGC 2264, which contains more than 360 near-IR sources (Lada et al. 1993; Lada \& Lada 2003). Located in the Mon OB1 molecular cloud complex $(d \sim 800$ pc), NGC 2264 is known for its famous Cone Nebula. Three arcminutes or $\sim 0.7$ pc to the north of the Cone Nebula, Allen (1972) discovered a bright embedded IR source, hereafter called IRS1, associated with IRAS 06384+0932, and also known as Allen's source. IRS1 is a B2-type object with estimated visual extinction $A_{\mathrm{V}} \sim 20-30$ (Allen 1972; Thompson et al. 1998), bolometric luminosity $L_{\text {bol }} \sim 2300 L_{\odot}$ (Margulis et al. 1989), and mass $M_{\star} \sim 9.5 M_{\odot}$ (Thompson et al. 1998). Approximatively 6 arcmin or $\sim 1.4 \mathrm{pc}$ to the north-west of IRS1, there is another IRAS source, IRAS 06382+0939, hereafter called IRS2, which is a Class I young stellar object (YSO) with $L_{\text {bol }} \sim 150 L_{\odot}$ (Margulis et al. 1989). Both IRS1 and IRS2 are associated with molecular outflows, named NGC 2264-C and NGC 2264-D, respectively (Margulis et al. 1988), and to dense molecular clumps revealed by $\mathrm{CS}(2-1)$ (Wolf-Chase et al. 1995) and submillimeter dust continuum observations (Ward-Thompson et al. 2000; Williams \& Garland 2002; Wolf-Chase et al. 2003). An $\mathrm{H}_{2} \mathrm{O}$ maser was found in each clump (Genzel et al. 1977; Mendoza et al. 1990), and a 44-GHz methanol maser was found in NGC 2264-C (Haschick et al. 1990). Methanol masers are known to be clear signposts of ongoing intermediate/high-mass star formation (Minier et al. 2001).

Previous kinematical studies of NGC 2264-C have revealed a complex velocity structure (Krügel et al. 1987). Evidence of large-scale collapse in the entire clump, disrupted by outflow motions on small scales, was reported by
Williams \& Garland (2002). Triggered star formation, due to an expanding shell $\sim 0.12 \mathrm{pc}$ in diameter around IRS1, has been suggested by Nakano et al. (2003) based on observations with the Nobeyama millimeter interferometer. These previous studies had either lower angular resolution and/or were focused on a much smaller region than the observations reported here.

The layout of the paper is as follows. Observational details, dust continuum results, and molecular line results are described in Sects. 2-4, respectively. Section 5 discusses the nature of the embedded millimeter sources identified in NGC 2264, while Sects. 6 and 7 present radiative transfer models of NGC 2264-C and NGC 2264-D-MM1, respectively. We compare our observational results with various scenarios of high-mass star formation in Sect. 8. Our main conclusions are summarized in Sect. 9.

\section{Millimeter observations}

We performed $1.2 \mathrm{~mm}$ dust continuum mapping observations of NGC 2264 in December 2000 with the IRAM 30 m telescope near Pico Veleta, Spain, using the MAMBO 37-channel bolometer array. Eleven on-the-fly maps were taken in the dual-beam scanning mode, with individual sizes ranging from $13^{\prime} \times 10^{\prime}$ to $5^{\prime} \times 4^{\prime}$. Chopping was performed by wobbling the secondary mirror of the telescope at $2 \mathrm{~Hz}$ with a throw ranging from $32^{\prime \prime}$ to $72^{\prime \prime}$. The scanning speed was either $6^{\prime \prime} / \mathrm{s}$ or $8^{\prime \prime} / \mathrm{s}$. The atmospheric opacity at zenith varied between 0.11 and 0.45 at $\lambda=1.25 \mathrm{~mm}$. Pointing and focus checks were made every hour. The pointing accuracy was measured to be better than 4 ", and the angular resolution was $\sim 11^{\prime \prime}(H P B W)$. Calibration was achieved by mapping Mars (primary calibrator) and is believed to be reliable to better than $\sim 15 \%$. Our resulting $1.2 \mathrm{~mm}$ continuum mosaic of the NGC 2264 region covers a total area of $\sim 400 \mathrm{arcmin}^{2}$, i.e, $\sim 23 \mathrm{pc}^{2}$ with a mean rms noise $\sigma=7 \mathrm{mJy} / 11^{\prime \prime}$-beam (i.e. $8 \mathrm{mJy} / 13^{\prime \prime}$-beam, see Fig. 1).

We performed follow-up molecular line observations with the IRAM $30 \mathrm{~m}$ telescope in March and May 2002. The observed molecular transitions were $\mathrm{N}_{2} \mathrm{H}^{+}(1-0), \mathrm{C}^{34} \mathrm{~S}(2-1)$, $\mathrm{HCO}^{+}(1-0), \mathrm{H}^{13} \mathrm{CO}^{+}(1-0)$ at $3 \mathrm{~mm}, \mathrm{CS}(3-2), \mathrm{C}^{34} \mathrm{~S}(3-2)$ at $2 \mathrm{~mm}, \mathrm{CS}(5-4), \mathrm{C}^{34} \mathrm{~S}(5-4), \mathrm{HCO}^{+}(3-2)$ and $\mathrm{H}^{13} \mathrm{CO}^{+}(3-2)$ at $1.1 \mathrm{~mm}$. The half-power beam width of the telescope was $\sim 26^{\prime \prime}, \sim 17^{\prime \prime}, \sim 10^{\prime \prime}$ at $3 \mathrm{~mm}, 2 \mathrm{~mm}, 1.1 \mathrm{~mm}$, respectively. For most transitions, we took maps in the on-the-fly mode around the peaks seen in the $1.2 \mathrm{~mm}$ dust continuum map of Fig. 1. We used either four SIS heterodyne receivers simultaneously or, for the $1.2 \mathrm{~mm}$ band, the HERA 9-pixel heterodyne array. As backend we used an autocorrelation spectrometer with a spectral resolution of $20 \mathrm{kHz}$ at $3 \mathrm{~mm}$ and of $40 \mathrm{kHz}$ at $2 \mathrm{~mm}$ and $1.2 \mathrm{~mm}$. The corresponding velocity resolution ranged from 0.05 to $0.08 \mathrm{~km} \mathrm{~s}^{-1}$ per channel.

\section{Dust continuum mapping results}

\subsection{Source extraction}

Our $1.2 \mathrm{~mm}$ dust continuum maps were reduced and combined with the IRAM software for bolometer-array data ("NIC"; cf. Broguière et al. 1995). Combining all our maps, we obtained the image shown in Fig. 1. The resulting mosaic 


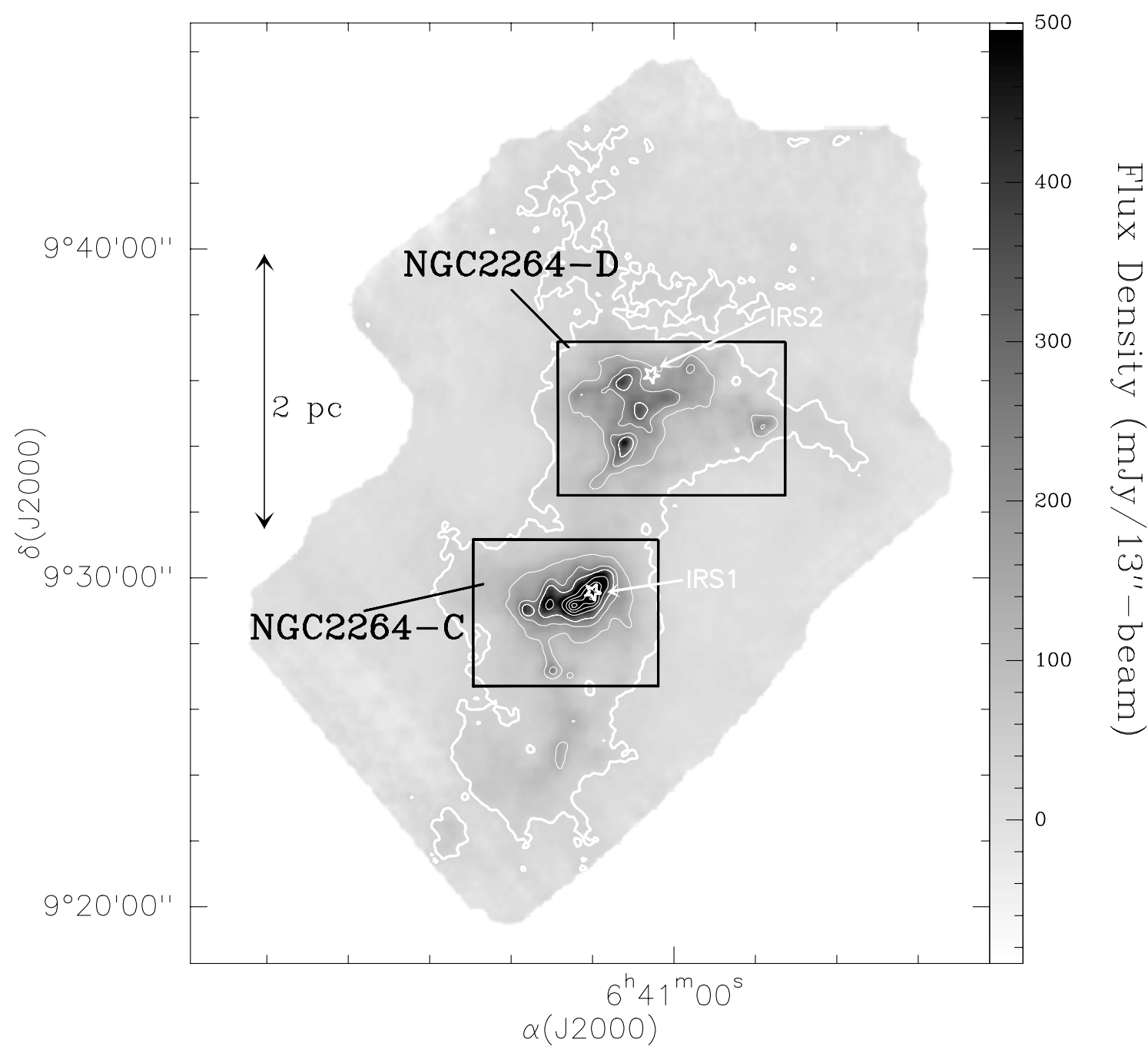

Fig. 1. Millimeter dust continuum mosaic of NGC 2264-C and NGC 2264-D, smoothed to an effective angular resolution of 13". The first white contour level is at $30 \mathrm{mJy} / 13^{\prime \prime}$-beam and corresponds to a column density $\sim 2 \times 10^{22} \mathrm{~cm}^{-2}$ (assuming $\kappa=0.005 \mathrm{~cm}^{2} \mathrm{~g}^{-1}$ and $T_{\mathrm{d}}=15 \mathrm{~K}$ ). The other white contour levels go from 120 to $300 \mathrm{mJy} / 13^{\prime \prime}$-beam by $90 \mathrm{mJy} / 13^{\prime \prime}$-beam, and from 300 to $1100 \mathrm{mJy} / 13^{\prime \prime}$-beam by $200 \mathrm{mJy} / 13^{\prime \prime}$-beam. The mean rms noise is $\sim 8 \mathrm{mJy} / 13^{\prime \prime}$-beam. The open white star symbols show the positions of the two IRAS sources IRS1 and IRS2.

confirms the presence of two distinct clumps, NGC 2264-C and NGC 2264-D, which closely follow the CS(2-1) integrated intensity map of Wolf-Chase et al. (1995).

In order to search for compact millimeter continuum sources (hereafter, MMSs) within NGC 2264-C and NGC 2264-D, we used the systematic method developed by Motte et al. (2003). This method is based on a combination of the multi-resolution wavelet algorithm of Starck et al. (1995) and the Gaussian fitting procedure of Stutzki \& Güsten (1990), Gaussclump (kindly provided by C. Kramer). The original image was first decomposed into two wavelet "views" of the field on small and large spatial scales, respectively. The difficult step at this stage is to select a proper limiting scale, called $\mathrm{ScUp}$, beyond which the detected dust emission is deemed to arise from the ambient cloud rather than from prestellar and/or protostellar sources. Here, we chose ScUp by analogy with the previous detailed study of the nearby $\rho$ Ophiuchi protocluster by Motte et al. (1998 - hereafter MAN98). In $\rho$ Ophiuchi, the physical diameter of dense cores (such as, e.g., Oph-A) was found to be $\sim 0.1 \mathrm{pc}$, corresponding to an angular diameter $\sim 24^{\prime \prime}$ at $800 \mathrm{pc}$. As the mean angular separation between nearest neighboring peaks in our dust continuum map of NGC 2264-C is also 24 ", we adopted ScUp $=24$ " in NGC 2264-C. In order to facilitate the comparison of source masses at the same spatial scale in the entire NGC 2264 region, we adopted ScUp $=24$ " in NGC 2264-D as well, even though the mean angular separation between nearest neighboring peaks is slightly larger $\left(\sim 40^{\prime \prime}\right)$ in clump D. The wavelet decomposition then allows us to filter out all emission structures arising from spatial scales larger than ScUp (see Figs. 2b and 3b).

In a second step, we used Gaussclump (Stutzki \& Güsten 1990) to identify all Gaussian sources with larger FWHM sizes than the $11^{\prime \prime}$ beam width and above a given peak flux density threshold in the filtered image (see Motte et al. 2003 for details). Following this procedure with the detection threshold set to $5 \sigma=35 \mathrm{mJy} / 11^{\prime \prime}$-beam, we identified a total of 27 MMSs: 12 in NGC 2264-C (see Fig. 2b) and 15 in NGC 2264-D (see Fig. 3b), 14 of which are new $1.2 \mathrm{~mm}$ 


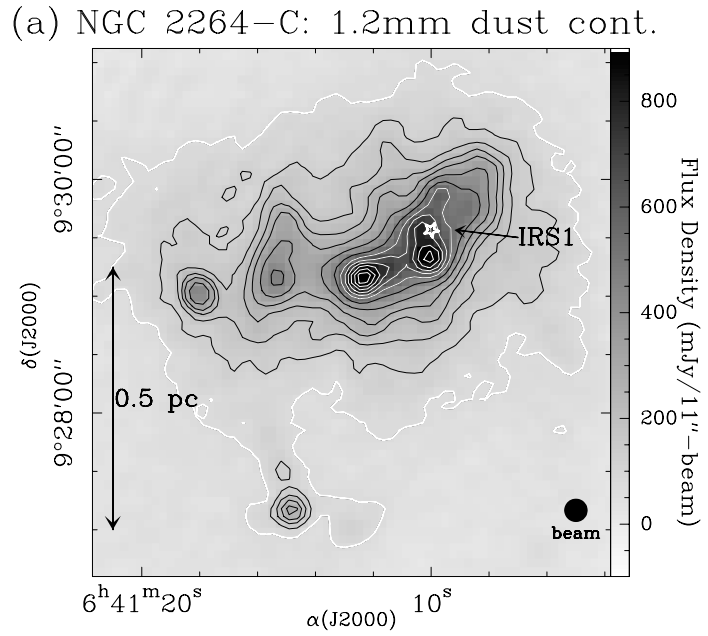

(b) NGC 2264-C: $1.2 \mathrm{~mm}$ filtered

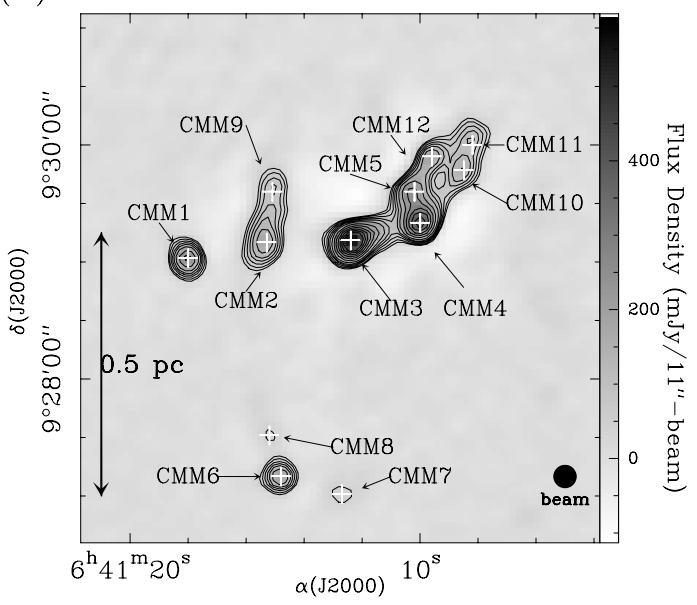

Fig. 2. a) Dust continuum map of NGC 2264-C at $1.2 \mathrm{~mm}$. The white open star shows the position of IRS1. The outer white contour corresponds to $S_{\text {peak }}^{1.2}=70 \mathrm{mJy} / 11^{\prime \prime}$-beam (i.e. $10 \sigma$ ), equivalent to a column density $\sim 7 \times 10^{22} \mathrm{~cm}^{-2}$ (assuming $\kappa=0.005 \mathrm{~cm}^{2} \mathrm{~g}^{-1}$ and $T_{\mathrm{d}}=$ $15 \mathrm{~K}$ ); the black contours go from 120 to 270 by $50 \mathrm{mJy} / 11^{\prime \prime}$-beam and from 350 to 450 by $100 \mathrm{mJy} / 11^{\prime \prime}$-beam, while the inner white contours go from 550 to 1050 by $100 \mathrm{mJy} / 11^{\prime \prime}$-beam. b) Filtered $1.2 \mathrm{~mm}$ dust continuum image of NGC 2264-C, obtained from the wavelet plane corresponding to spatial scales smaller than ScUp $=24^{\prime \prime}$. The white crosses mark the central positions of the MMSs identified with Gaussclump. The contours go from 35 to 50 by $15 \mathrm{mJy} / 11^{\prime \prime}$-beam, from 50 to 110 by $20 \mathrm{mJy} / 11^{\prime \prime}$-beam and from 140 to 440 by $50 \mathrm{mJy} / 11^{\prime \prime}$-beam (see text for details).

dust continuum detections. Table 1 lists the parameters of the 27 detected Gaussian MMSs. Although three more peaks lie just above $35 \mathrm{mJy} /$ beam in the filtered map of NGC 2264-D (Fig. 3b), they are not identified as real MMSs, since their $F W H M$ sizes are smaller than the beam width.

\subsection{Properties of the NGC 2264 sources}

Assuming optically thin $1.2 \mathrm{~mm}$ dust continuum emission, the measured flux densities provide direct estimates of the masses and column densities of the MMSs (see, e.g., MAN98 for details). We adopted a dust mass opacity $\kappa_{\text {dust }}=0.005 \mathrm{~cm}^{2} \mathrm{~g}^{-1}$ at $1.2 \mathrm{~mm}$ (cf. MAN98) and a dust temperature $T_{\mathrm{d}}=15 \mathrm{~K}$ (a) NGC 2264-D: 1.2mm dust cont.
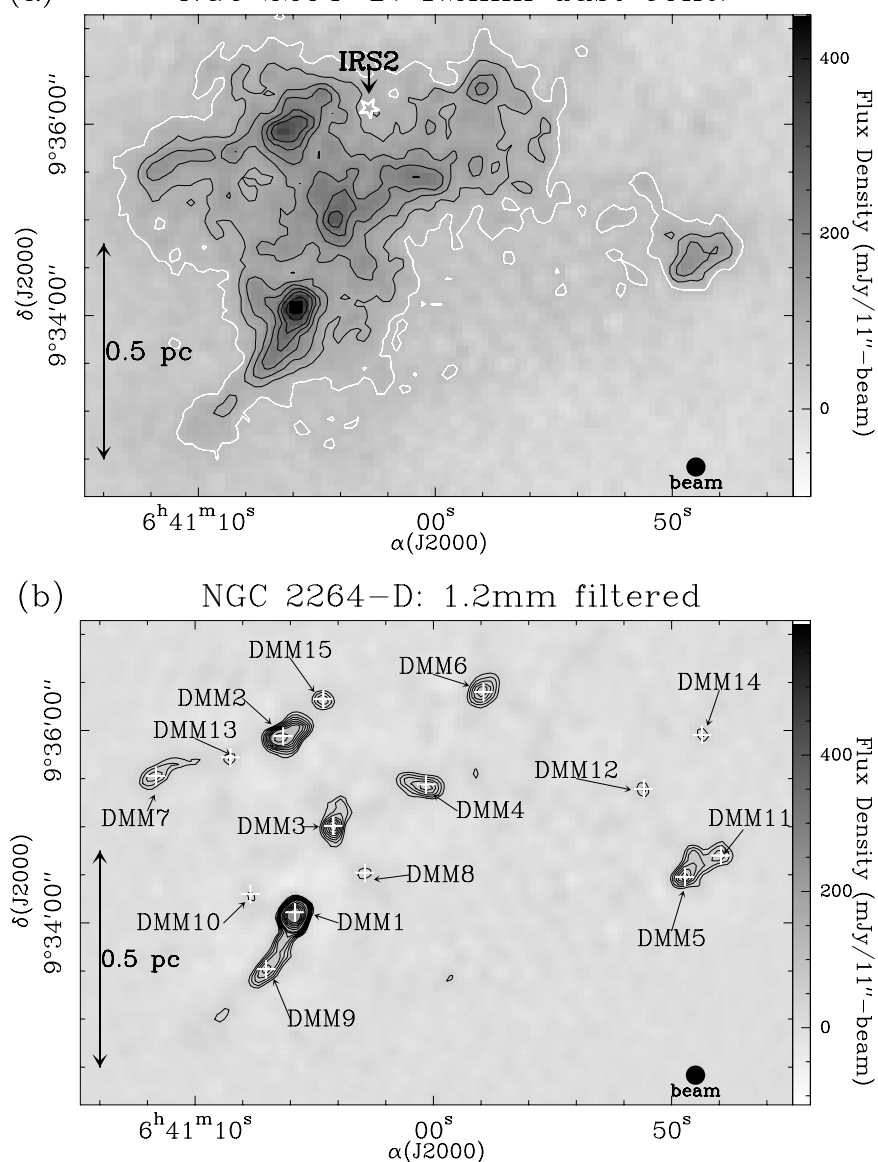

Fig. 3. Same as Fig. 2 for NGC 2264-D. a) The white open star shows the position of IRS2. The outer white contour is the same as in Fig. 2a, while the other black contours go from 110 to 270 by $40 \mathrm{mJy} / 11^{\prime \prime}$-beam and from 350 to 425 by $75 \mathrm{mJy} / 11^{\prime \prime}$-beam. b) The contours go from 35 to 95 by $10 \mathrm{mJy} / 11^{\prime \prime}$-beam and from 120 to 200 by $20 \mathrm{mJy} / 11^{\prime \prime}$-beam (see text for details).

(Ward-Thompson et al. 2000) for all sources. The distance to NGC 2264 was taken to be $d=800 \mathrm{pc}$ and the mean molecular weight $\mu=2.33$. The derived masses, column densities, and volume densities are listed in Table 2 for both NGC 2264-C and NGC 2264-D. Note that the masses of the compact MMSs account for only $\sim 10 \%$ of the total gas mass of the clumps, with the latter dominated by the large-scale background.

Given the Gaussian fit uncertainties, a source is considered as unresolved if its undeconvolved $F W H M$ angular size is smaller than $13^{\prime \prime}$. Four sources were found to be unresolved (with both the major and minor FWHM sizes smaller than $13^{\prime \prime}$ ); twelve are only partly resolved (with only the minor FWHM smaller than $13^{\prime \prime}$ ), and eleven are fully resolved (with both the major and minor FWHMs larger than $13^{\prime \prime}$ ). The MMSs have deconvolved $F W H M$ physical sizes ranging from $<0.03 \mathrm{pc}$ to $0.12 \mathrm{pc}$, masses ranging from $\sim 2$ to $\sim 41 M_{\odot}$, and volume densities ranging from $\sim 1.5 \times 10^{5}$ to $3 \times 10^{6} \mathrm{~cm}^{-3}$; we can only estimate lower limits to the volume densities of the unresolved MMSs. Our $5 \sigma$ detection threshold corresponds to a mass of $\sim 1.2 M_{\odot}$. A detailed comparison of the global properties of NGC 2264-C and NGC 2264-D is presented in Sect. 8 
Table 1. Source extraction results in NGC 2264-C and NGC 2264-D.

\begin{tabular}{|c|c|c|c|c|c|c|c|}
\hline \multirow[t]{2}{*}{ Source $^{a}$} & \multicolumn{2}{|c|}{ Coordinates } & \multirow{2}{*}{$\begin{array}{c}\text { Undec. } F W H M^{b} \\
\text { (arcsecond) }\end{array}$} & \multirow{2}{*}{$\begin{array}{l}\mathrm{PA}^{c} \\
(\mathrm{deg})\end{array}$} & \multirow{2}{*}{$\begin{array}{c}S_{\text {peak }}^{1.2 d} \\
\left(\mathrm{mJy} / 11^{\prime \prime} \text {-beam) }\right.\end{array}$} & \multirow{2}{*}{$\begin{array}{l}\begin{array}{l}S_{\text {int }}^{1.2 e} \\
(\mathrm{mJy})\end{array} \\
\end{array}$} & \multirow{2}{*}{$\begin{array}{c}S_{\text {back }}^{1.2 f} \\
\left(\mathrm{mJy} / 11^{\prime \prime} \text {-beam) }\right.\end{array}$} \\
\hline & $\alpha_{2000}$ & $\delta_{2000}$ & & & & & \\
\hline C-MM1 & $06: 41: 18.0$ & $+09: 29: 02$ & $14 \times 12$ & 16 & $255 \pm 11$ & 354 & 186 \\
\hline C-MM2 & $06: 41: 15.3$ & $+09: 29: 10$ & $22 \times 13$ & -12 & $183 \pm 10$ & 433 & 296 \\
\hline C-MM3 & $06: 41: 12.4$ & $+09: 29: 11$ & $18 \times 13$ & 123 & $573 \pm 9$ & 1108 & 508 \\
\hline C-MM4 & $06: 41: 10.0$ & $+09: 29: 20$ & $18 \times 15$ & -11 & $426 \pm 8$ & 951 & 541 \\
\hline C-MM5 & $06: 41: 10.2$ & $+09: 29: 36$ & $21 \times 11$ & 135 & $261 \pm 9$ & 498 & 529 \\
\hline C-MM6 & $06: 41: 14.8$ & $+09: 27: 10$ & $12 \times 12$ & - & $183 \pm 6$ & 218 & 100 \\
\hline C-MM7 & $06: 41: 12.7$ & $+09: 27: 01$ & $15 \times 13$ & 126 & $45 \pm 6$ & 73 & 68 \\
\hline C-MM8 & $06: 41: 15.2$ & $+09: 27: 31$ & $14 \times 11$ & 23 & $35 \pm 6$ & 45 & 89 \\
\hline C-MM9 & $06: 41: 15.1$ & $+09: 29: 36$ & $21 \times 11$ & -1 & $94 \pm 10$ & 179 & 225 \\
\hline C-MM10 & $06: 41: 08.5$ & $+09: 29: 47$ & $27 \times 12$ & -24 & $140 \pm 8$ & 375 & 366 \\
\hline C-MM11 & $06: 41: 08.2$ & $+09: 30: 00$ & $16 \times 11$ & 103 & $110 \pm 7$ & 160 & 288 \\
\hline C-MM12 & 06:41:09.8 & $+09: 29: 51$ & $14 \times 11$ & 121 & $183 \pm 7$ & 233 & 387 \\
\hline D-MM1 & $06: 41: 05.8$ & $+09: 34: 06$ & $17 \times 13$ & -24 & $257 \pm 8$ & 469 & 211 \\
\hline D-MM2 & $06: 41: 06.3$ & $+09: 35: 56$ & $23 \times 14$ & 116 & $134 \pm 9$ & 357 & 204 \\
\hline D-MM3 & $06: 41: 04.2$ & $+09: 35: 01$ & $18 \times 12$ & -11 & $105 \pm 9$ & 187 & 201 \\
\hline D-MM4 & $06: 41: 00.3$ & $+09: 35: 26$ & $26 \times 13$ & 81 & $81 \pm 9$ & 226 & 159 \\
\hline D-MM5 & $06: 40: 49.4$ & $+09: 34: 29$ & $19 \times 13$ & 135 & $88 \pm 8$ & 180 & 93 \\
\hline D-MM6 & $06: 40: 57.9$ & $+09: 36: 24$ & $20 \times 15$ & -39 & $86 \pm 9$ & 213 & 119 \\
\hline D-MM7 & $06: 41: 11.6$ & $+09: 35: 32$ & $28 \times 13$ & 126 & $60 \pm 8$ & 180 & 116 \\
\hline D-MM8 & $06: 41: 02.9$ & $+09: 34: 31$ & $15 \times 11$ & 33 & $41 \pm 9$ & 56 & 136 \\
\hline D-MM9 & $06: 41: 07.0$ & $+09: 33: 31$ & $31 \times 12$ & 139 & $77 \pm 7$ & 237 & 137 \\
\hline D-MM10 & $06: 41: 07.7$ & $+09: 34: 18$ & $16 \times 11$ & 12 & $36 \pm 8$ & 52 & 136 \\
\hline D-MM11 & $06: 40: 47.9$ & $+09: 34: 42$ & $19 \times 15$ & 109 & $62 \pm 8$ & 146 & 90 \\
\hline D-MM12 & $06: 40: 51.2$ & $+09: 35: 24$ & $12 \times 11$ & 51 & $49 \pm 10$ & 53 & 53 \\
\hline D-MM13 & $06: 41: 08.5$ & $+09: 35: 43$ & $12 \times 11$ & -34 & $46 \pm 8$ & 50 & 137 \\
\hline D-MM14 & $06: 40: 48.7$ & $+09: 35: 57$ & $12 \times 11$ & 29 & $46 \pm 10$ & 50 & 26 \\
\hline D-MM15 & 06:41:04.6 & $+09: 36: 19$ & $16 \times 12$ & 102 & $62 \pm 9$ & 98 & 146 \\
\hline
\end{tabular}

a The C-MM and D-MM numbers correspond to our own labelling and are consistent with the source numbering of Ward-Thompson et al. (2000) and Wolf-Chase et al. (2003) for previously known sources.

${ }^{b}$ Undeconvolved $F W H M$ sizes derived from fitting an elliptical Gaussian to the background subtracted maps (i.e., after filtering out emission seen on scales larger than $\mathrm{ScUp}=24^{\prime \prime}$ ).

${ }^{c}$ Position angle (from North to East) of the major axis of the fitted Gaussian ellipse.

${ }^{d}$ Peak flux density in the background subtracted continuum map and $1 \sigma \mathrm{rms}$ at the position of the source.

$e$ Total integrated flux density under the elliptical Gaussian.

${ }^{f}$ Background level at the position of the source peak.

and Table 6 below. Here, we simply note that NGC 2264-C appears to be more centrally concentrated than NGC 2264-D in Fig. 1.

\section{Molecular line-mapping results}

\section{1. $\mathrm{N}_{2} \mathrm{H}^{+}$line widths and velocity dispersion}

Maps in low optical depth tracers of dense gas, such as $\mathrm{N}_{2} \mathrm{H}^{+}$, can provide constraints on the velocity field of protoclusters projected onto the line of sight (cf. Belloche et al. 2001). The $\mathrm{N}_{2} \mathrm{H}^{+}$molecule, which does not deplete up to fairly high densities (e.g. Bergin \& Langer 1997; Belloche \& André 2004), is a particularly interesting tracer in this respect. Figures 4 and 5 show that the $\mathrm{N}_{2} \mathrm{H}^{+}(1-0)$ integrated intensity maps of NGC 2264-C and NGC 2264-D essentially trace the same structures as the $1.2 \mathrm{~mm}$ dust continuum maps when the latter are smoothed to the same 27" angular resolution.

We used the HFS (HyperFine Structure) fitting routine of the CLASS reduction package from IRAM to fit all 7 hyperfine components of the $\mathrm{N}_{2} \mathrm{H}^{+}(1-0)$ multiplet at each mapped position. This provided, for each spectrum, estimates of the centroid velocity, $V_{\mathrm{LSR}}$, and of the $F W H M$ linewidth, $\Delta V$.

The $\mathrm{N}_{2} \mathrm{H}^{+}$linewidth measured toward each MMS is listed as a velocity dispersion $\sigma_{\text {line }}=\Delta V / \sqrt{8 \ln (2)}$ in Table 2 . 
Table 2. Properties ${ }^{a}$ of the millimeter sources detected in NGC 2264-C and NGC 2264-D.

\begin{tabular}{|c|c|c|c|c|c|c|c|c|}
\hline Source & $\begin{array}{c}\text { Dec. } F W H M^{b} \\
(\mathrm{pc})\end{array}$ & $\begin{array}{l}M_{1.2^{c}} \\
\left(M_{\odot}\right)\end{array}$ & $\begin{array}{c}\text { Density }^{d} \\
\left(\mathrm{~cm}^{-3}\right)\end{array}$ & $\begin{array}{c}N_{\mathrm{H}_{2}}{ }^{e} \\
\left(10^{22} \mathrm{~cm}^{-2}\right)\end{array}$ & $\begin{array}{c}N_{\mathrm{H}_{2}}^{\text {back } f} \\
\left(10^{22} \mathrm{~cm}^{-2}\right) \\
\end{array}$ & $\begin{array}{c}\sigma_{\text {line }^{g}} \\
\left(\mathrm{~km} \mathrm{~s}^{-1}\right)\end{array}$ & $\begin{array}{l}M_{\mathrm{vir}}{ }^{h} \\
\left(M_{\odot}\right) \\
\end{array}$ & $\overline{\alpha_{\mathrm{vir}}{ }^{i}}$ \\
\hline C-MM1 & $0.035 \times \mathrm{UR}$ & 13.1 & $1.8 \times 10^{6}$ & 26 & 19 & 0.38 & 3.2 & 0.2 \\
\hline C-MM2 & $0.076 \times 0.028$ & 16.0 & $6.7 \times 10^{5}$ & 18 & 30 & 0.51 & 8.4 & 0.5 \\
\hline C-MM3 & $0.057 \times 0.028$ & 40.9 & $2.7 \times 10^{6}$ & 57 & 51 & 1.06 & 31.3 & 0.8 \\
\hline C-MM4 & $0.057 \times 0.041$ & 35.1 & $1.3 \times 10^{6}$ & 43 & 54 & 0.89 & 26.7 & 0.8 \\
\hline C-MM5 & $0.072 \times \mathrm{UR}$ & 18.4 & $8.4 \times 10^{5}$ & 26 & 53 & 0.93 & 27.1 & 1.5 \\
\hline C-MM6 & unresolved & 8.1 & $>1.5 \times 10^{6}$ & 18 & 10 & 0.34 & 2.3 & 0.3 \\
\hline C-MM7 & $0.041 \times 0.028$ & 2.7 & $2.9 \times 10^{5}$ & 5 & 7 & 0.59 & 8.2 & 3.0 \\
\hline C-MM8 & $0.035 \times \mathrm{UR}$ & 1.7 & $2.3 \times 10^{5}$ & 4 & 9 & 0.47 & 4.8 & 2.8 \\
\hline C-MM9 & $0.072 \times \mathrm{UR}$ & 6.6 & $3.0 \times 10^{5}$ & 9 & 23 & 0.55 & 9.5 & 1.4 \\
\hline C-MM10 & $0.099 \times \mathrm{UR}$ & 13.9 & $3.9 \times 10^{5}$ & 14 & 37 & 0.76 & 21.2 & 1.5 \\
\hline C-MM11 & $0.046 \times \mathrm{UR}$ & 5.9 & $5.3 \times 10^{5}$ & 11 & 29 & 0.68 & 11.6 & 2.0 \\
\hline C-MM12 & $0.035 \times \mathrm{UR}$ & 8.6 & $1.2 \times 10^{6}$ & 18 & 39 & 0.76 & 12.6 & 1.5 \\
\hline D-MM1 & $0.052 \times 0.028$ & 17.3 & $1.3 \times 10^{6}$ & 26 & 21 & 0.59 & 9.3 & 0.5 \\
\hline D-MM2 & $0.081 \times 0.035$ & 13.2 & $3.6 \times 10^{5}$ & 13 & 20 & 0.51 & 9.7 & 0.7 \\
\hline D-MM3 & $0.057 \times \mathrm{UR}$ & 6.9 & $4.5 \times 10^{5}$ & 11 & 20 & 0.26 & 1.9 & 0.3 \\
\hline D-MM4 & $0.094 \times 0.028$ & 8.4 & $2.6 \times 10^{5}$ & 8 & 16 & 0.81 & 23.5 & 2.8 \\
\hline D-MM5 & $0.062 \times 0.028$ & 6.7 & $3.8 \times 10^{5}$ & 9 & 9 & 0.42 & 5.1 & 0.8 \\
\hline D-MM6 & $0.067 \times 0.041$ & 7.9 & $2.3 \times 10^{5}$ & 9 & 12 & 0.55 & 11.0 & 1.4 \\
\hline D-MM7 & $0.103 \times 0.028$ & 6.7 & $1.8 \times 10^{5}$ & 6 & 12 & 0.59 & 13.0 & 1.9 \\
\hline D-MM8 & $0.041 \times \mathrm{UR}$ & 2.1 & $2.2 \times 10^{5}$ & 4 & 14 & 0.34 & 2.7 & 1.3 \\
\hline D-MM9 & $0.116 \times \mathrm{UR}$ & 8.9 & $2.0 \times 10^{5}$ & 8 & 14 & 0.55 & 12.0 & 1.3 \\
\hline D-MM10 & $0.046 \times \mathrm{UR}$ & 1.9 & $1.5 \times 10^{5}$ & 4 & 14 & 0.72 & 13.0 & 6.8 \\
\hline D-MM11 & $0.062 \times 0.041$ & 5.4 & $1.7 \times 10^{5}$ & 6 & 9 & 0.30 & 3.2 & 0.6 \\
\hline D-MM12 & unresolved & 1.9 & $>3.6 \times 10^{5}$ & 5 & 5 & 0.38 & 2.8 & 1.5 \\
\hline D-MM13 & unresolved & 1.8 & $>3.4 \times 10^{5}$ & 5 & 14 & 0.89 & 15.5 & 8.6 \\
\hline D-MM14 & unresolved & 1.8 & $>3.4 \times 10^{5}$ & 5 & 3 & - & - & - \\
\hline D-MM15 & $0.046 \times \mathrm{UR}$ & 3.6 & $3.2 \times 10^{5}$ & 6 & 15 & 0.64 & 10.2 & 2.8 \\
\hline
\end{tabular}

a All parameters have been estimated from the background-subtracted maps, i.e., after filtering out emission seen on scales larger than $\mathrm{ScUp}=24^{\prime \prime}($ see text)

${ }^{b}$ Deconvolved major and minor $F W H M$ continuum sizes; UR means unresolved.

${ }^{c}$ Mass estimated from the total $1.2 \mathrm{~mm}$ continuum flux density of the corresponding elliptical Gaussian source. For a 2D Gaussian source, $\sim 94 \%$ of this mass is contained within an area twice the size of the FWHM ellipse. Typical uncertainty is a factor $\gtrsim 2$ (on either side) due to the uncertain values of the dust mass opacity and dust temperature.

${ }^{d}$ Mean $\mathrm{H}_{2}$ volume density derived from the mass of Col. 3 assuming a spherical source of effective diameter twice the geometrical mean of the deconvolved $F W H M$ sizes in Col. 2. Typical uncertainty is a factor $\gtrsim 2$ as for $M_{1.2}$. The mean density within the $F W H M$ volume is four times larger.

e Peak column density estimated from the background-subtracted map. Typical uncertainty is a factor $\gtrsim 2$ as for $M_{1.2}$.

${ }^{f}$ Background column density at the position of the source peak assuming the same dust properties as for the MMSs (see text).

$g$ Velocity dispersion (i.e. rms) along the line of sight derived from a Gaussian hyperfine fit to the $\mathrm{N}_{2} \mathrm{H}^{+}(1-0)$ multiplet. The typical fit uncertainty is $\Delta \sigma_{\text {line }}=0.04 \mathrm{~km} \mathrm{~s}^{-1}$.

${ }^{h}$ Virial mass calculated as $M_{\text {vir }}=3 R \frac{\sigma_{\text {line }}^{2}}{G}$ assuming a $\rho \propto r^{-2}$ density profile. Here, the radius $R$ is equal to twice the geometrical mean of the deconvolved $H W H M$ radii. Typical relative uncertainty is less than $30 \%$.

${ }^{i}$ Virial parameter defined as $\alpha_{\mathrm{vir}}=M_{\mathrm{vir}} / \mathrm{M}_{1.2}$. Typical uncertainty is a factor $\gtrsim 2$, dominated by the uncertainty on $M_{1.2}$.

On average, we found $\sigma_{\text {line }} \sim 0.6-0.7 \mathrm{~km} \mathrm{~s}^{-1}$ in both NGC 2264-C and NGC 2264-D. From these $\sigma_{\text {line }}$ estimates, we can calculate a virial mass, $M_{\mathrm{vir}}$, and a virial mass ratio (cf. Bertoldi \& McKee 1992), $\alpha_{\text {vir }}=M_{\text {vir }} / M_{1.2}$, for each MMS (cf. Table 2). Note that the $\mathrm{N}_{2} \mathrm{H}^{+}(1-0)$ observations probe material on a $27^{\prime \prime}$ scale, while the typical FWHM size of the dust continuum sources is only $\sim 15^{\prime \prime}$. However, since both $M_{\text {vir }}$ and $M_{1.2}$ are derived for a diameter $\sim$ twice the $F W H M$ size, our method of estimating $\alpha_{\text {vir }}$ should be reliable. Given the observational uncertainties on $M_{\mathrm{vir}}$ and $M_{1.2}$, most MMSs are consistent with 


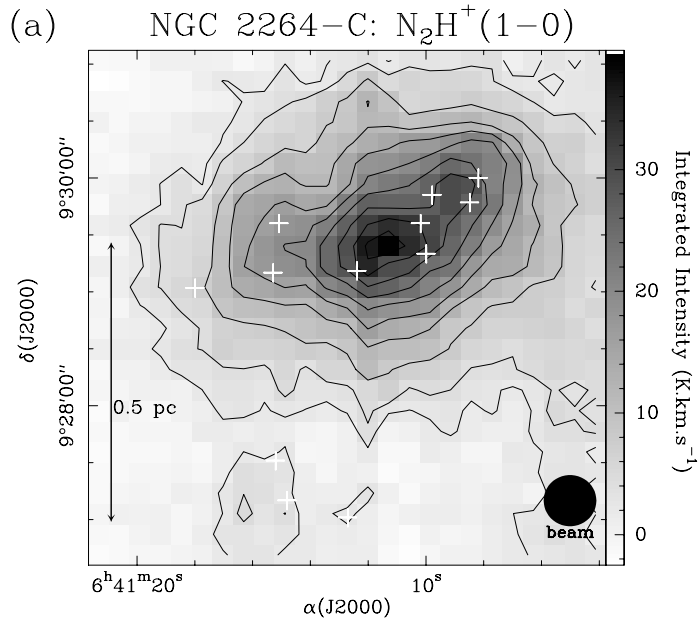

(b) NGC 2264-C: 1.2mm smoothed

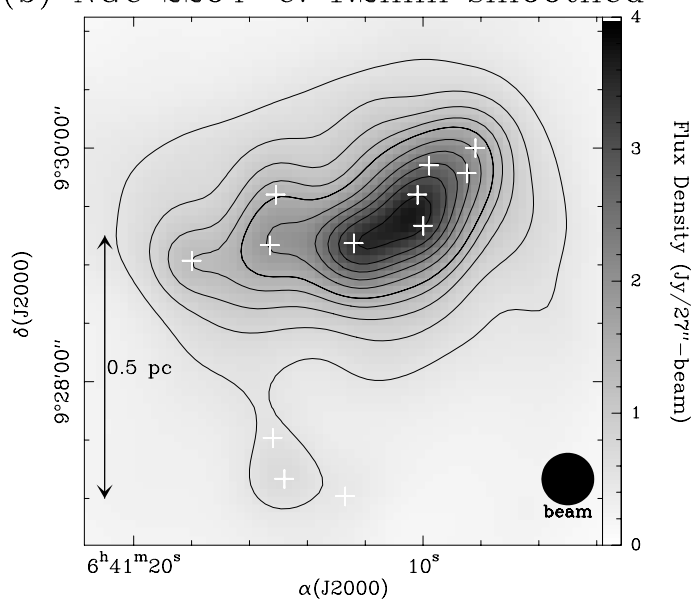

Fig. 4. a) Map of the $\mathrm{N}_{2} \mathrm{H}^{+}(1-0)$ integrated line intensity from 0 to $25 \mathrm{~km} \mathrm{~s}^{-1}$ in NGC 2264-C. First contour at $2 \mathrm{~K} \mathrm{~km} \mathrm{~s}^{-1}$; other contours go from 4 to $36 \mathrm{~K} \mathrm{~km} \mathrm{~s}^{-1}$ by $4 \mathrm{~K} \mathrm{~km} \mathrm{~s}^{-1}$. b) Millimeter dust continuum map of NGC 2264-C smoothed to the same 27" $(H P B W)$ angular resolution as the $\mathrm{N}_{2} \mathrm{H}^{+}(1-0)$ map. Contour levels go from 0.5 to $1.5 \mathrm{Jy} / 27^{\prime \prime}$-beam by $0.25 \mathrm{Jy} / 27^{\prime \prime}$-beam and from 1.5 to $3 \mathrm{Jy} / 27^{\prime \prime}$-beam by $0.375 \mathrm{Jy} / 27^{\prime \prime}$-beam. The white crosses mark the positions of the millimeter continuum sources identified in Sect. 3.

virial equilibrium (i.e., $\alpha_{\text {vir }} \sim 1$ ). This is discussed further in Sect. 8. The measured values of $\sigma_{\text {line }}$ are to be compared with the thermal sound speed, $c_{\mathrm{s}}$, which is $\sim 0.23 \mathrm{~km} \mathrm{~s}^{-1}$ for molecular gas at $15 \mathrm{~K}$. All MMSs have $\sigma_{\text {line }}>\mathrm{c}_{\mathrm{s}}$, indicating that supersonic turbulence still dominates thermal broadening down to spatial scales $\leq 0.1 \mathrm{pc}$ in the NGC 2264 region $^{1}$. The typical Mach number $M=\sqrt{3} \frac{\sigma}{c_{s}}$ of the gas within the MMSs is larger than 5. On the larger $\sim 0.5-1$ pc spatial scale of the NGC 2264 clumps, we estimated the Mach number to be $M \gtrsim 7$, based on the linewidth of the mean $\mathrm{N}_{2} \mathrm{H}^{+}(1-0)$ spectrum averaged over the whole extent of NGC 2264-C and NGC 2264-D, respectively (cf. Table 3). We conclude that NGC 2264 is

\footnotetext{
${ }^{1}$ In some cases, gravitational collapse may contribute to the linewidth, so the value of $M_{\text {vir }}$ quoted in Table 2 may be correspondingly overestimated. The radiative transfer models we present in Sects. 6 and 7 below suggest that infall contributes $\sim 20-50 \%$ of the linewidth for C-MM3 and D-MM1.
}
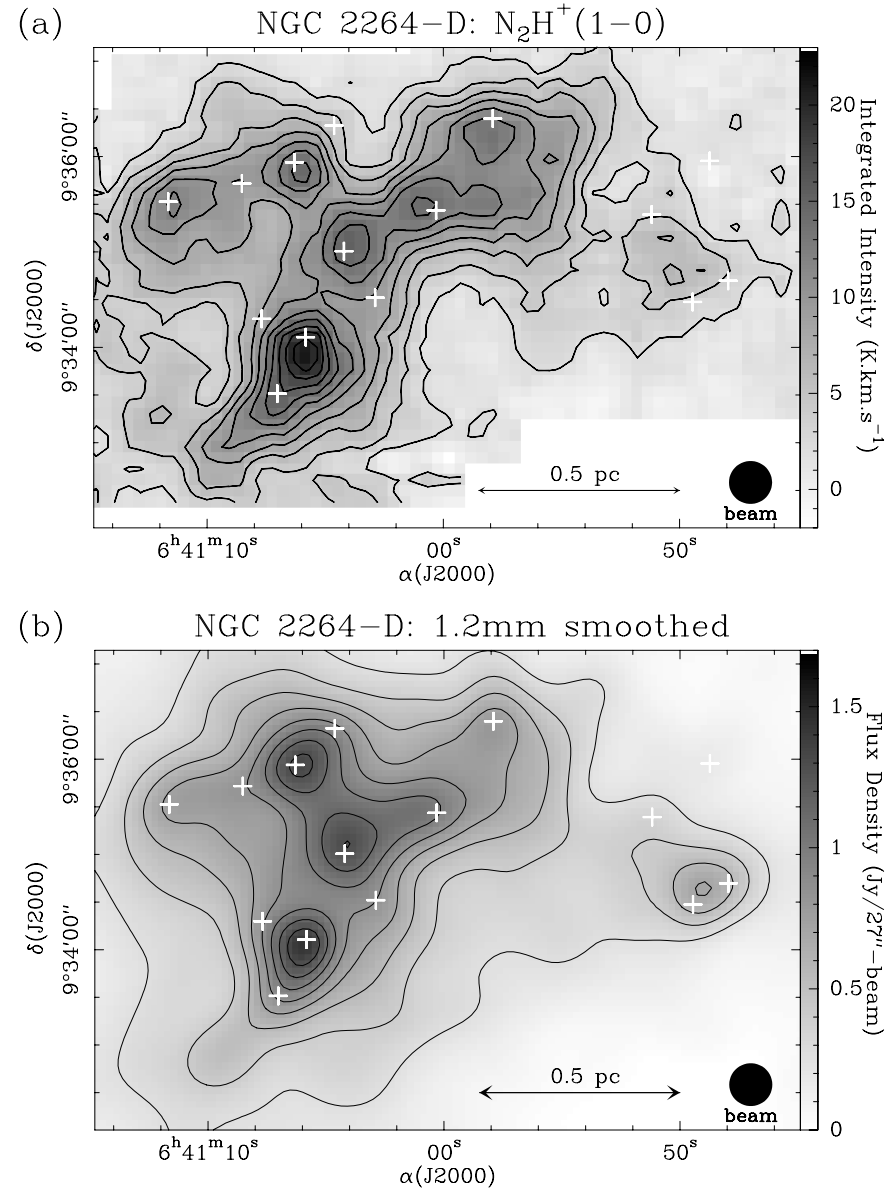

Fig. 5. Same as Fig. 4 for NGC 2264-D. a) Contour levels go from 2 to $20 \mathrm{~K} \mathrm{~km} \mathrm{~s}^{-1}$ by $2 \mathrm{~K} \mathrm{~km} \mathrm{~s}^{-1}$. b) Contour levels go from 0.25 to $1.3 \mathrm{Jy} / 27^{\prime \prime}$-beam by $0.15 \mathrm{Jy} / 27^{\prime \prime}$-beam.

Table 3. Mean $\mathrm{N}_{2} \mathrm{H}^{+}(1-0)$ line properties of clumps $\mathrm{C}$ and D.

\begin{tabular}{ccccc}
\hline \hline Clump & $\begin{array}{c}V_{\text {sys }}{ }^{a} \\
\left(\mathrm{~km} \mathrm{~s}^{-1}\right)\end{array}$ & $\begin{array}{c}\left\langle\sigma_{\text {line }}{ }^{b}\right. \\
\left(\mathrm{km} \mathrm{s}^{-1}\right)\end{array}$ & $\begin{array}{c}\sigma_{1 \mathrm{D}}{ }^{c} \\
\left(\mathrm{~km} \mathrm{~s}^{-1}\right)\end{array}$ & $\begin{array}{c}\sigma_{3 \mathrm{D}}{ }^{d} \\
\left(\mathrm{~km} \mathrm{~s}^{-1}\right)\end{array}$ \\
\hline NGC 2264-C & $7.5 \pm 0.2$ & $1.1 \pm 0.1$ & $0.7 \pm 0.1$ & $1.3 \pm 0.3$ \\
NGC 2264-D & $5.5 \pm 0.2$ & $1.0 \pm 0.1$ & $0.8 \pm 0.2$ & $1.4 \pm 0.3$ \\
\hline
\end{tabular}

${ }^{a}$ Mean systemic velocity and rms dispersion around that value.

${ }^{b}$ Mean $\mathrm{N}_{2} \mathrm{H}^{+}(1-0)$ line-of-sight velocity dispersion averaged over each clump and rms dispersion around that value.

${ }^{c}$ Standard deviation of the distribution of source centroid velocities measured in each clump. The error bar has been estimated as $\sqrt{\frac{2}{n-1}} \frac{\sigma_{1 \mathrm{D}}}{2}$, assuming that the source sample is drawn from a larger population whose velocity distribution follows Gaussian statistics.

${ }^{d} 3 \mathrm{D}$ velocity dispersion of the MMSs calculated from $\sigma_{1 \mathrm{D}}$ assuming isotropic motions. The error bar has been scaled from that estimated for $\sigma_{1 \mathrm{D}}$.

characterized by supersonic motions down to the spatial scale of the MMSs.

We could also estimate the dispersion, $\sigma_{1 \mathrm{D}}$, of the lineof-sight velocities of the MMSs relative to one another in each clump. Assuming isotropic motions, we could infer the 3D velocity dispersion of the MMSs within each 
clump, $\sigma_{3 \mathrm{D}}=\sqrt{3} \sigma_{1 \mathrm{D}}$ (see Table 3 ). Based on these velocity dispersion estimates, the crossing times of the MMSs across their parent clumps were calculated to be $t_{\text {cross }} \sim 6.0 \times 10^{5} \mathrm{yr}$ in Clump C and $\sim 6.3 \times 10^{5}$ yr in Clump D.

\subsection{A velocity discontinuity at the center of NGC 2264-C}

Our mapping in low-optical depth transitions (e.g. $\mathrm{N}_{2} \mathrm{H}^{+}(1-0)$ and $\left.\mathrm{H}^{13} \mathrm{CO}^{+}(1-0)\right)$ revealed a remarkable velocity discontinuity in the innermost part of NGC 2264-C near the position of the continuum source C-MM3. This is illustrated in Fig. 6, which shows $\mathrm{N}_{2} \mathrm{H}^{+}(1-0)$ and $\mathrm{H}^{13} \mathrm{CO}^{+}(1-0)$ positionvelocity (PV) diagrams observed along four different axes. In the PV diagrams taken along the East-West (EW) axis going through the continuum sources C-MM2, C-MM3, and C-MM4 (top two panels of Fig. 6), one can clearly see two distinct velocity components associated with C-MM2 and C-MM4 at $\sim 6.5 \mathrm{~km} \mathrm{~s}^{-1}$ and $\sim 8.5 \mathrm{~km} \mathrm{~s}^{-1}$, respectively, both extending over more than $50^{\prime \prime}$ (i.e., $\sim 0.2 \mathrm{pc}$ ). These two velocity components overlap at the position of C-MM3, forming a sharp velocity discontinuity $\sim 2 \mathrm{~km} \mathrm{~s}^{-1}$ in amplitude. The other panels of Fig. 6 show PV diagrams taken along North-South axes at three different right-ascension positions, i.e., passing through C-MM2 (labelled NS-2 axis), C-MM3 (NS-3 axis), and C-MM4 (NS-4 axis), respectively. Along the NS-4 axis, only the higher velocity component at $\sim 8.5 \mathrm{~km} \mathrm{~s}^{-1}$ can be seen, while along the NS-2 axis only the lower velocity component at $\sim 6.5 \mathrm{~km} \mathrm{~s}^{-1}$ is visible. Both velocity components are visible in the PV diagram along the NS-3 axis.

We stress that the velocity feature seen in Fig. 6 cannot be explained by rotation. Indeed, both velocity components are strong at the center of the system (i.e. C-MM3) with little emission at intermediate velocities, while the opposite trend would be expected in the case of rotation. Moreover, the rotational curve expected from differential rotation is characterized by a continuous "S" shape (cf. Belloche et al. 2002) rather than a sharp velocity discontinuity as observed here.

Finally, we note that no similar velocity discontinuity exists at the center of the NGC 2264-D clump.

\section{3. $\mathrm{HCO}^{+}(3-2)$ signatures of infall and outflow}

Our extensive $\mathrm{HCO}^{+}(3-2)$ maps of both NGC 2264 clumps are shown in Fig. 7, in the form of spectra overlaid on the $1.2 \mathrm{~mm}$ dust continuum images. The $\mathrm{HCO}^{+}(3-2)$ transition, which is optically thick and often self-absorbed in dense cores, is a good tracer of inward/outward motions (e.g. Evans 1999) when associated with an optically thin tracer such as $\mathrm{H}^{13} \mathrm{CO}^{+}(3-2)$. A double-peaked $\mathrm{HCO}^{+}(3-2)$ spectrum with a blue peak that is stronger than the red peak is usually taken to be a diagnosis of infall motions, provided that the corresponding optically thin $\mathrm{H}^{13} \mathrm{CO}^{+}(3-2)$ spectrum peaks in the dip of the $\mathrm{HCO}^{+}(3-2)$ line profile. Conversely, an $\mathrm{HCO}^{+}(3-2)$ spectrum skewed to the red, e.g., with a red peak stronger than the blue peak, leads to a diagnosis of outflow motions. These diagnoses are valid only for centrally-condensed sources (such as dense cores), as they assume that the line excitation temperature increases toward source center. In NGC 2264-C and NGC 2264-D, a variety of $\mathrm{HCO}^{+}(3-2)$ line shapes were observed (cf. Fig. 7), ranging from clear "blue" infall profiles toward C-MM3 and D-MM1, to typical "red" outflow profiles toward C-MM4 and D-MM3. Examples of unclear, mixed profiles were observed toward C-MM2 or D-MM2. This variety of $\mathrm{HCO}^{+}(3-2)$ line shapes suggests that the velocity field within the NGC 2264 clumps is complex and possibly results from a combination of small-scale inflows/outflows directly associated with the MMSs and larger-scale systematic motions in the entire clumps. This will be discussed in Sects. 6 and 7 below.

\section{Nature of the embedded sources of NGC 2264}

We classified the extracted MMSs as "protostellar" or "prestellar" based on the presence or absence of an outflow, jet or embedded YSO signature (see Tables 4 and 5). For this purpose, we used existing outflow/jet studies of the NGC 2264 region (Margulis et al. 1988; Schreyer et al. 1997, 2003; Wang et al. 2002), as well as the extensive 2MASS and MSX near-/mid-IR surveys. Our classification of the NGC 2264-D sources relied entirely on comparison with the 2MASS and MSX surveys, as no systematic jet survey was available for clump D. By default, MMSs with no IR counterparts in NGC 2264-D are tentatively classified as "prestellar", even though we cannot rule out that some may be Class 0 protostellar sources.

We found that up to $\sim 70 \%$ of the MMSs in clump C and at least $25 \%$ of the MMSs in clump D are protostellar in nature. Although we generally lacked good bolometric luminosity estimates for the protostellar MMSs and thus cannot prove that their submillimeter to bolometric luminosity ratios match the definition of Class 0 protostars (André et al. 1993), that they exhibit strong millimeter continuum emission suggests that they all are good candidate Class 0 objects. We note that the Class 0 nature of one of the protostellar MMSs, D-MM1, has been reliably established on the basis of SCUBA imaging and HIRES processing of the IRAS data (Wolf-Chase et al. 2003).

The apparent difference in protostellar fraction between clump C and clump D may be partly due to the lack of outflow/jet survey in clump D. As an illustration, C-MM1 and C-MM2 in clump C are both classified as protostars, because they show evidence of jets, while they are not associated with any near-/mid-IR sources. The high fraction of protostars found in NGC 2264-C contrasts with the significantly lower $\sim 40 \%$ fraction of protostellar sources observed by MAN98 in $\rho$-Oph. It is also noteworthy that the protostellar sources of NGC 2264-C are all concentrated in the dense inner ridge visible in Fig. 3a, while the prestellar sources are all located in the outskirts of clump C.

When comparing our present observations of NGC 2264 with the study of MAN98 in $\rho$ Oph, one should keep in mind that there is a difference of a factor $\sim 5$ in distance, hence in effective spatial resolution. Based on the $1.2 \mathrm{~mm}$ continuum mosaic of MAN98, if $\rho$-Oph were at the same distance as NGC 2264 (800 pc), our source extraction method would only have detected four sources (above the same detection threshold as in NGC 2264), corresponding to the prominent dense cores 

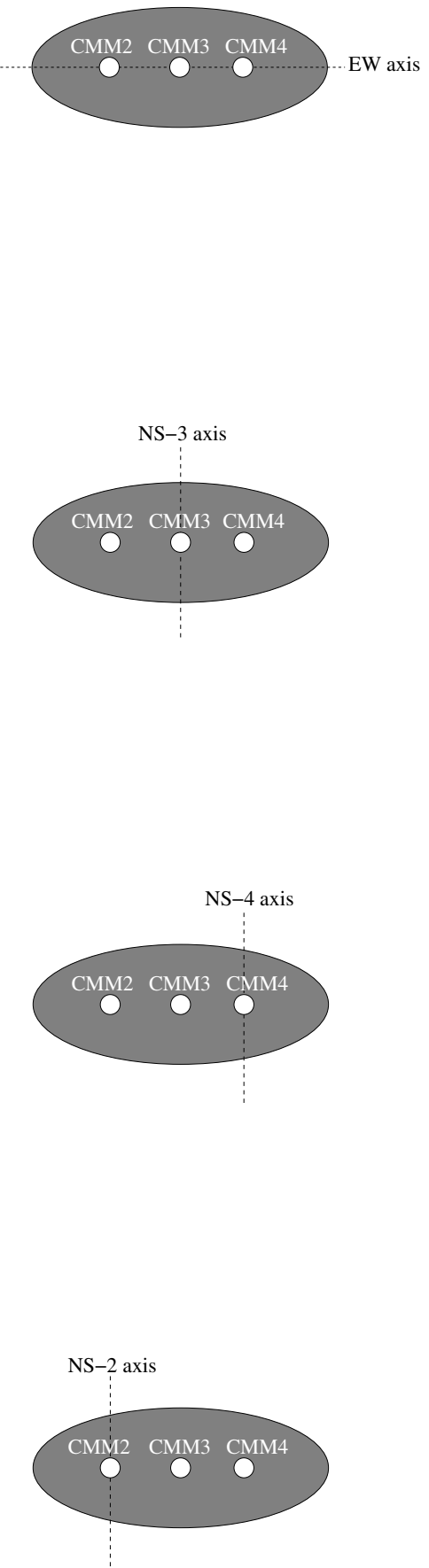

$\mathrm{N}_{2} \mathrm{H}^{+}(101-012)$ position-velocity diagrams

$\mathrm{H}^{13} \mathrm{CO}^{+}(1-0)$ position-velocity diagrams

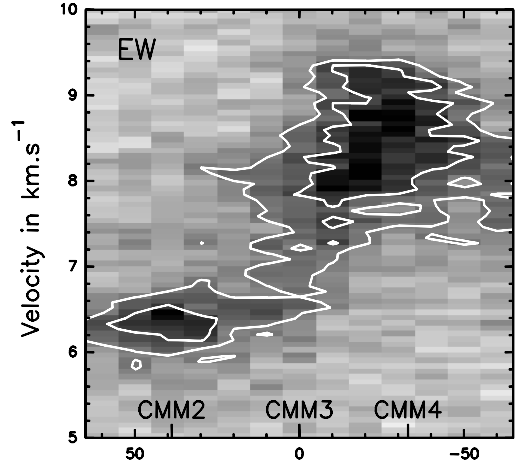

Position in arcsecond along EW axis
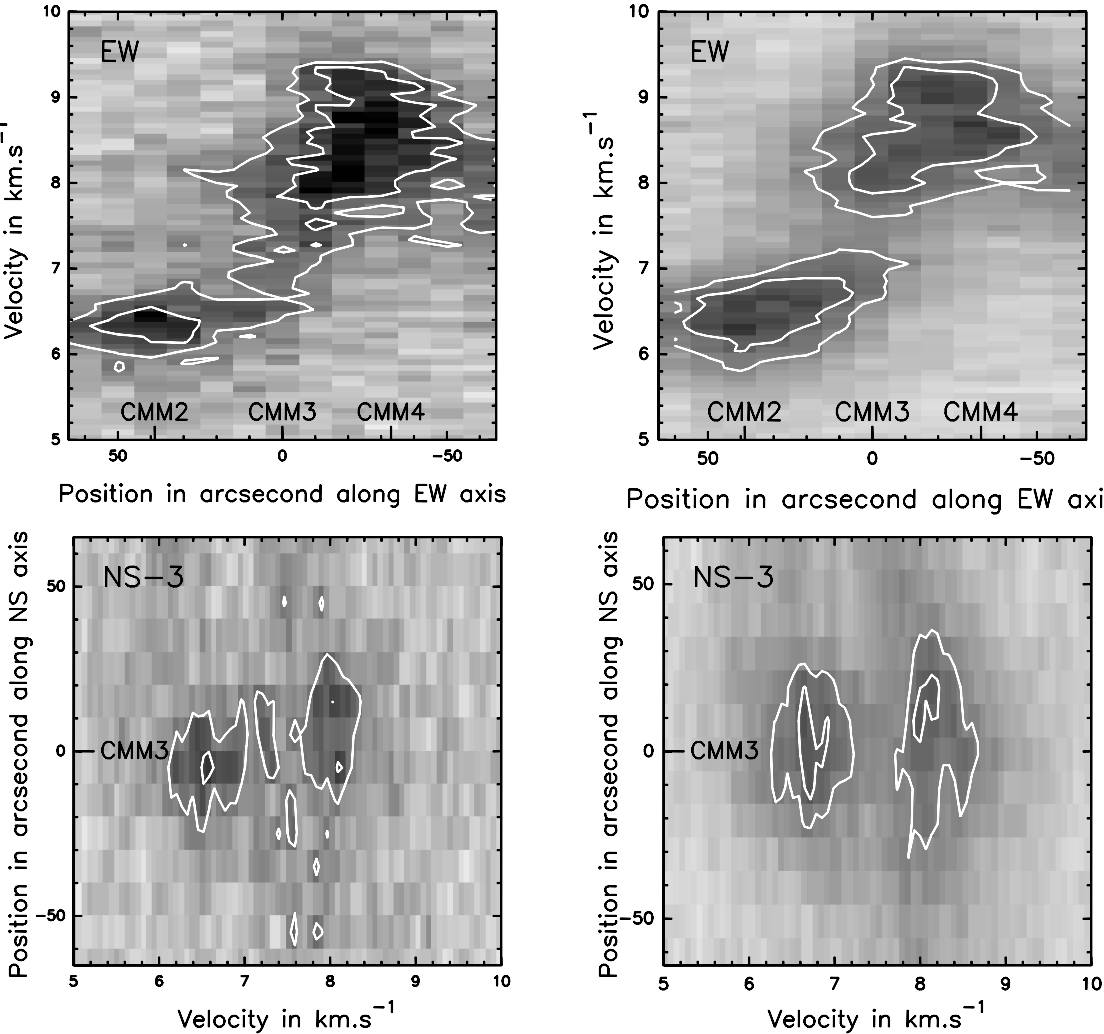

Position in arcsecond along EW axis
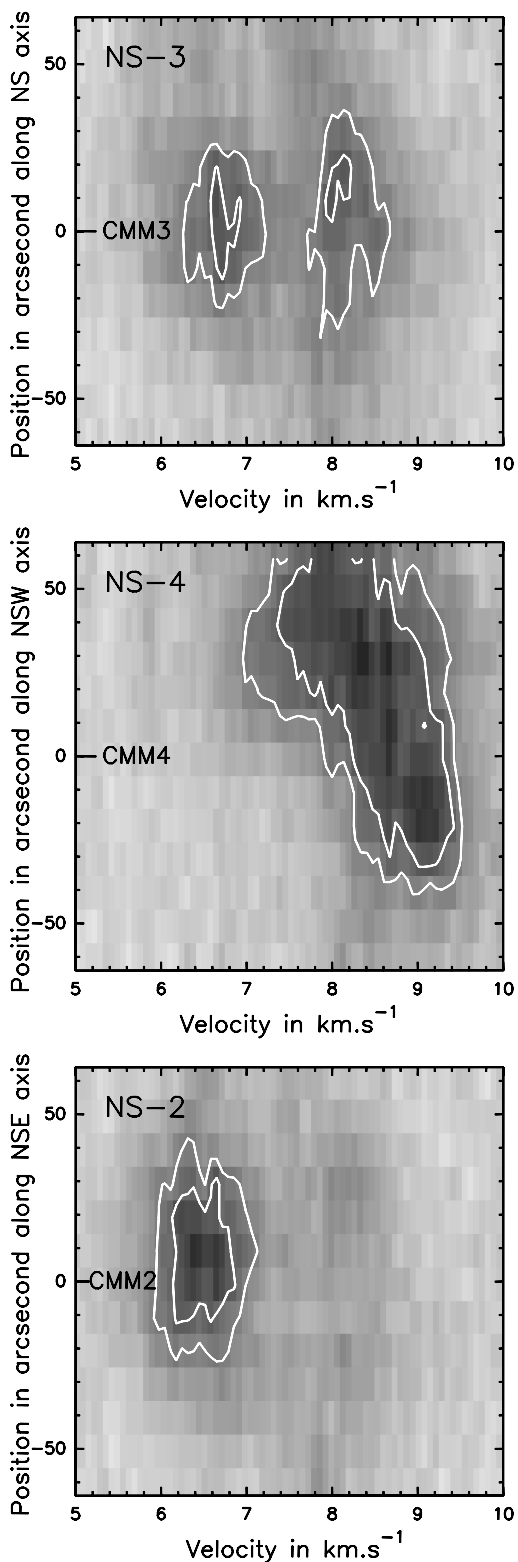

Fig. 6. $\mathrm{N}_{2} \mathrm{H}^{+}(101-012)$ and $\mathrm{H}^{13} \mathrm{CO}^{+}(1-0)$ position-velocity diagrams observed in the central part of NGC 2264-C along four axes shown schematically on the left. From top to bottom: the first row corresponds to PV diagrams taken along an East-West (EW) axis going through C-MM2, C-MM3, and C-MM4; the second row corresponds to a North-South axis going through C-MM3 (NS-3); the third row is for a NorthSouth axis going through C-MM4 (NS-4); the fourth row is for a North-South axis going through C-MM2 (NS-2). Contour levels are 1.1 and $1.8 \mathrm{~K}$ for the $\mathrm{N}_{2} \mathrm{H}^{+}(101-012)$ diagrams, 1.05 and $1.4 \mathrm{~K}$ for the $\mathrm{H}^{13} \mathrm{CO}^{+}(1-0)$ diagrams. 

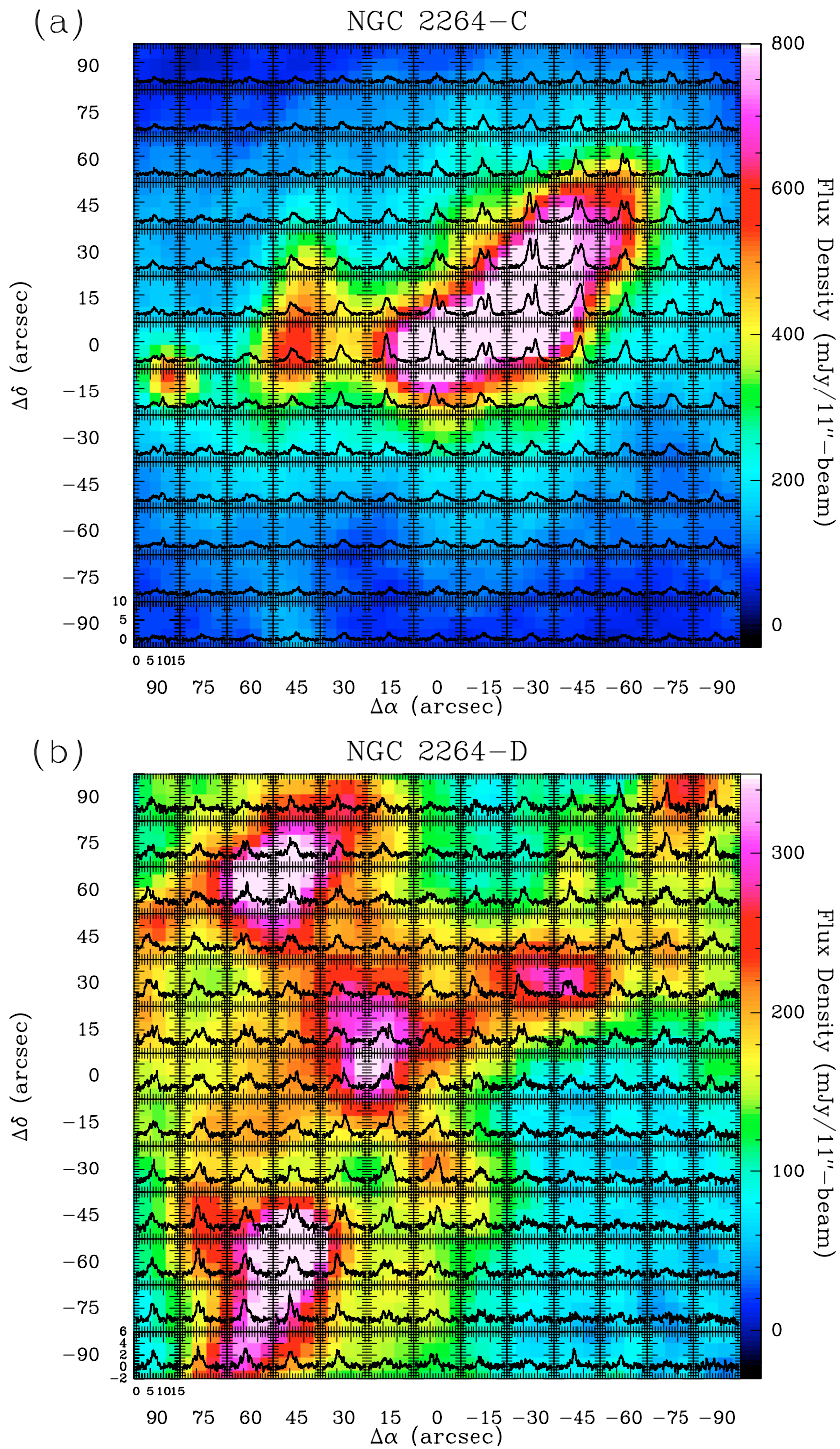

Fig. 7. a) Map of $\mathrm{HCO}^{+}(3-2)$ spectra observed toward NGC 2264-C, overlaid on the $1.2 \mathrm{~mm}$ dust continuum image (grey scale); the $(0,0)$ position corresponds to C-MM3. b) Same as (a) for NGC 2264-D; the $(0,0)$ position corresponds to D-MM3.

Oph A, Oph B2, Oph C, and Oph F (cf. Loren et al. 1990). We conclude that the MMSs identified here in NGC 2264 resemble the $\mathrm{DCO}^{+}$"dense cores" of $\rho$ Oph more than the compact starless condensations found by MAN98 (called "starless clumps" therein). This may partly explain why we find a larger fraction of protostellar sources in NGC 2264: each dense core in $\rho$-Oph harbors at least one protostar and several starless condensations. Observed from a distance of $800 \mathrm{pc}$, such a core would very likely be classified as "protostellar", even though the majority of its small-scale condensations actually are "prestellar".

On the other hand, millimeter continuum interferometer observations by Nakano et al. (2003) and Schreyer et al. (2003) show that the objects called C-MM4, C-MM5, and C-MM12 here remain unfragmented single sources at a spatial resolution of $23000 \mathrm{AU}$. This may suggest that the MMSs detected with the $30 \mathrm{~m}$ telescope in NGC 2264 are more centrally
Table 4. Nature of the millimeter continuum sources identified in NGC 2264-C.

\begin{tabular}{ccccc}
\hline \hline Source & MSX/2MASS $^{a}$ & $\mathrm{H}_{2}$ jet $^{b}$ & CS flow $^{c}$ & Nature $^{d}$ \\
\hline C-MM1 & 0 & $\mathrm{Y}$ & & pro \\
C-MM2 & 0 & $\mathrm{Y}$ & & pro \\
C-MM3 & 3 & $\mathrm{Y}$ & & pro \\
C-MM4 & 1 & $?$ & $\mathrm{~N}$ & pro \\
C-MM5 & 2 & $?$ & $\mathrm{~N}$ & pro \\
C-MM6 & 0 & $\mathrm{~N}$ & & pre \\
C-MM7 & 0 & $\mathrm{~N}$ & & pre \\
C-MM8 & 0 & $\mathrm{~N}$ & & pre \\
C-MM9 & 0 & $\mathrm{~N}$ & & pre \\
C-MM10 & 1 & $\mathrm{~N}$ & $\mathrm{Y}$ & pro \\
C-MM11 & 2 & $\mathrm{~N}$ & & pro \\
C-MM12 & 2 & $?$ & $\mathrm{Y}$ & pro \\
\hline
\end{tabular}

a Number of 2MASS or MSX infrared sources lying within an $11^{\prime \prime}$ beam centered on the MMS position.

${ }^{b}$ Detection of a shocked $\mathrm{H}_{2}$ jet by Wang et al. (2002).

${ }^{c}$ Detection of a CS outflow by Schreyer et al. (2003).

$d$ "pro" stands for protostellar, "pre" for prestellar.

Table 5. Nature of the millimeter continuum sources identified in NGC 2264-D.

\begin{tabular}{ccc}
\hline \hline Source & MSX/2MASS $^{a}$ & Nature $^{b}$ \\
\hline D-MM1 & 1 & pro \\
D-MM2 & 0 & pre \\
D-MM3 & 1 & pro \\
D-MM4 & 0 & pre \\
D-MM5 & 0 & pre \\
D-MM6 & 0 & pre \\
D-MM7 & 1 & pro \\
D-MM8 & 0 & pre \\
D-MM9 & 0 & pre \\
D-MM10 & 0 & pre \\
D-MM11 & 0 & pre \\
D-MM12 & 0 & pre \\
D-MM13 & 0 & pre \\
D-MM14 & 1 & pro \\
D-MM15 & 0 & pre \\
\hline
\end{tabular}

${ }^{a}$ Number of 2MASS or MSX infrared sources lying within an $11^{\prime \prime}$ beam centered on the MMS position.

$b$ "pro" stands for protostellar, "pre" for prestellar.

concentrated than the $\rho$-Oph dense cores (see Sect. 8 for further comparisons).

The bright infrared source IRS1 lies close to, but is clearly offset from, C-MM5 (Nakano et al. 2002; Schreyer et al. 2003). Based on its slightly rising spectral energy distribution between $12 \mu \mathrm{m}$ and $100 \mu \mathrm{m}$, Margulis et al. (1989) has classified IRS 1 as a Class I object with $L_{\text {bol }}=2300 L_{\odot}$. The detections of 
VLA radio continuum emission (Schwartz et al. 1985), as well as $\mathrm{H}_{2} \mathrm{O}$ and methanol masers, at the position of IRS1 are suggestive of a $\sim 9.5 M_{\odot}$ YSO on the ZAMS (cf. Thompson et al. 1998). Although there is apparently neither disk nor outflow directly associated with it (Schreyer et al. 2003), IRS1 is thus clearly a relatively massive embbeded young star.

The other infrared source detected by IRAS in the region discussed in this paper is IRS2, which lies in clump D, just outside the densest region (see Figs. 1 and 13). It is significantly less luminous than IRS1 $\left(L_{\mathrm{bol}}=150 L_{\odot}-\right.$ Margulis et al. 1989) and is not closely associated with any millimeter continuum source. It has also been classified as a relatively young (Class I) object by Margulis et al. (1989).

\section{Radiative transfer modelling of NGC 2264-C}

Here, we compare our molecular line observations of NGC 2264-C with radiative transfer calculations performed with the same Monte-Carlo radiative transfer code as used by Belloche et al. (2002). This code is divided into two parts. The first part calculates the non-LTE level populations based on a 1D Monte-Carlo method (Bernes 1978, 1979). The second, called MAPYSO (Blinder 1997), integrates the radiative transfer equation along each line of sight and convolves the resulting data cube with the beam of the telescope. MAPYSO can be used in either $1 \mathrm{D}$ or $2 \mathrm{D}$ geometry. The $2 \mathrm{D}$ mode was used for the present modelling of NGC 2264-C. The inputs to the code are the density and abundance profiles, the kinetic temperature profile, the $2 \mathrm{D}$ velocity field, and the non-thermal velocity dispersion which, for simplicity, was assumed to be uniform over the simulated region.

The averaged radial density profile of NGC 2264-C can be inferred from the corresponding $1.2 \mathrm{~mm}$ dust continuum intensity profile $I(r)$, where $r$ is the spherical radius. The best powerlaw fit to the observed intensity profile is found to be $I(r) \propto$ $r^{-0.4}$. Assuming isothermal dust emission in the Rayleigh-Jeans regime, this translates into an average spherical density profile $\rho(r) \propto r^{-1.4}$ (cf. Motte \& André 2001), which has been normalized so as to yield a total mass of $1650 M_{\odot}$ within a radius of $0.4 \mathrm{pc}$, as observed (cf. Table 6 below). Since the projected center of gravity of the NGC 2264-C clump, as estimated from our dust continuum map, is located at the position of C-MM3, we centered our models on C-MM3 rather than C-MM4, as adopted by Williams \& Garland (2002).

The gas kinetic temperature profile was taken to be $T_{k}(r)=$ $\left(\left(T_{0}\left(\frac{r}{r_{0}}\right)^{-0.4}\right)^{4}+T_{1}^{4}\right)^{0.25}$ with $T_{0}=90 \mathrm{~K}, r_{0}=100 \mathrm{AU}$ and $T_{1}=$ $15 \mathrm{~K}$. The value of $T_{0}$ was adjusted so as to match the intensity of the observed spectra. This temperature profile is consistent with heating from a luminous central source $\left(L_{\mathrm{bol}} \sim 80 L_{\odot}\right)$ that is radiating in an optically thin medium and is embedded in a cold $15 \mathrm{~K}$ background at large radii.

The $\mathrm{CS}$ and $\mathrm{HCO}^{+}$molecules are known to be depleted onto dust grains above a density $n_{\mathrm{H} 2} \sim 10^{5} \mathrm{~cm}^{-3}$ in starless cores (e.g. Tafalla et al. 2002). The spherical abundance profiles were thus fixed to standard values, i.e. $[\mathrm{CS}] /\left[\mathrm{H}_{2}\right]=1 \times 10^{-9}$ (i.e. Tafalla et al. 2002) and $\left[\mathrm{HCO}^{+}\right] /\left[\mathrm{H}_{2}\right]=1.5 \times 10^{-9}$ (i.e. Bergin et al. 1997), in the low-density outer regions and were assumed to scale as $n_{\mathrm{H}_{2}}^{-0.4}$ with density (cf. Bacmann et al. 2002).

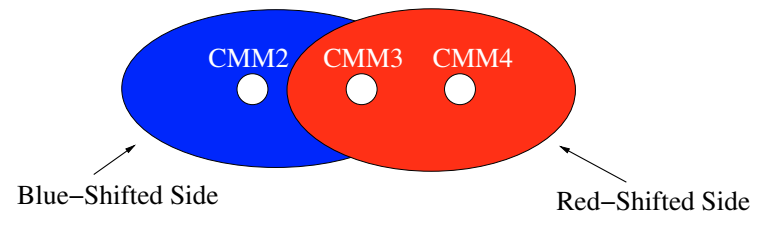

(a)

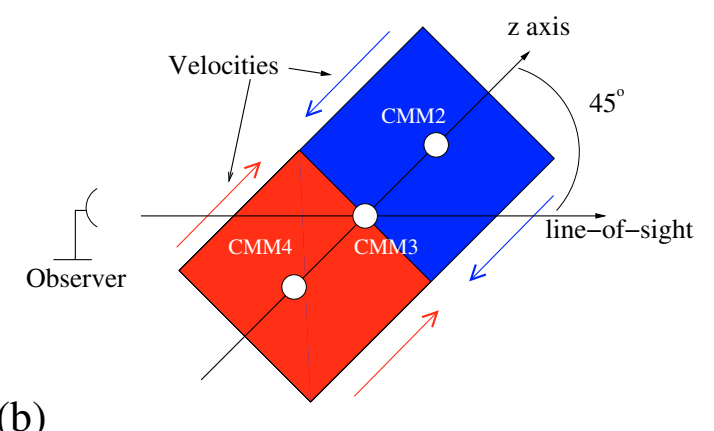

Fig. 8. a) Schematic view of our proposed model of NGC 2264-C as seen by an observer on the plane of the sky. b) Side view of the plane containing the $z$-axis and the line of sight: a finite-sized, cylindrical clump collapses toward its center, coinciding with C-MM3. The long axis (i.e. $z$-axis) of the cylinder makes an angle of 45 degrees with the line of sight.

We assumed the decrease in abundance with density to stop where the kinetic temperature rises above $20 \mathrm{~K}$ in the inner region (see Fig. 9). Still further in, where the gas becomes warmer than $\sim 50 \mathrm{~K}$, one may expect the abundances to increase as a result of grain mantle evaporation. However, $T_{k}$ reaches $50 \mathrm{~K}$ only at very small radii $\left(<1000 \mathrm{AU}\right.$ corresponding to $\left.<1^{\prime \prime}\right)$ in our model, and this small inner region has no influence on spectra observed at $>10^{\prime \prime}$ angular resolution. We also adopted constant, standard values of 22 and 75 for the $[\mathrm{CS}] /\left[\mathrm{C}^{34} \mathrm{~S}\right]$ and $\left[\mathrm{HCO}^{+}\right] /\left[\mathrm{H}^{13} \mathrm{CO}^{+}\right]$isotopic ratios, respectively. The nonthermal velocity dispersion was set to $\sigma_{\mathrm{NT}}=0.47 \mathrm{~km} \mathrm{~s}^{-1}$, i.e., slightly less than the $\mathrm{N}_{2} \mathrm{H}^{+}(1-0)$ linewidths measured in NGC 2264-C (cf. Sect. 4.1), which accounts for the contribution of collapse motions to the linewidth. Finally, the infall velocity profile was adjusted so as to fit the observed spectra.

In order to account for the velocity discontinuity observed in the central part of NGC 2264-C, we modelled the clump as a cylindrical filament centered on C-MM3 (see Fig. 8). The system was assumed to be collapsing toward its center of mass (i.e. C-MM3), so that the two sides of the filament (C-MM2 and C-MM4) are moving toward each other. The long axis of the filament was taken to be inclined by $45^{\circ}$ to the line-of-sight (see Fig. $8 \mathrm{~b}$ ). When probing the kinematics of such structure, one would indeed observe a sharp velocity discontinuity near the center of the filament. The velocity profile was taken to be only a function of $z$ (position along the long axis of the cylinder), with $v(z)=V_{\text {inf }}=1.3 \mathrm{~km} \mathrm{~s}^{-1}$ for $z<0$ and $v(z)=-V_{\text {inf }}$ for $z>0$ in the inner $|z|<35000 \mathrm{AU}$ (or 45") region, and sharp, symmetrical decreases of $|v(z)|$ with $|z|$ outside the central region (cf. Fig. 9). 


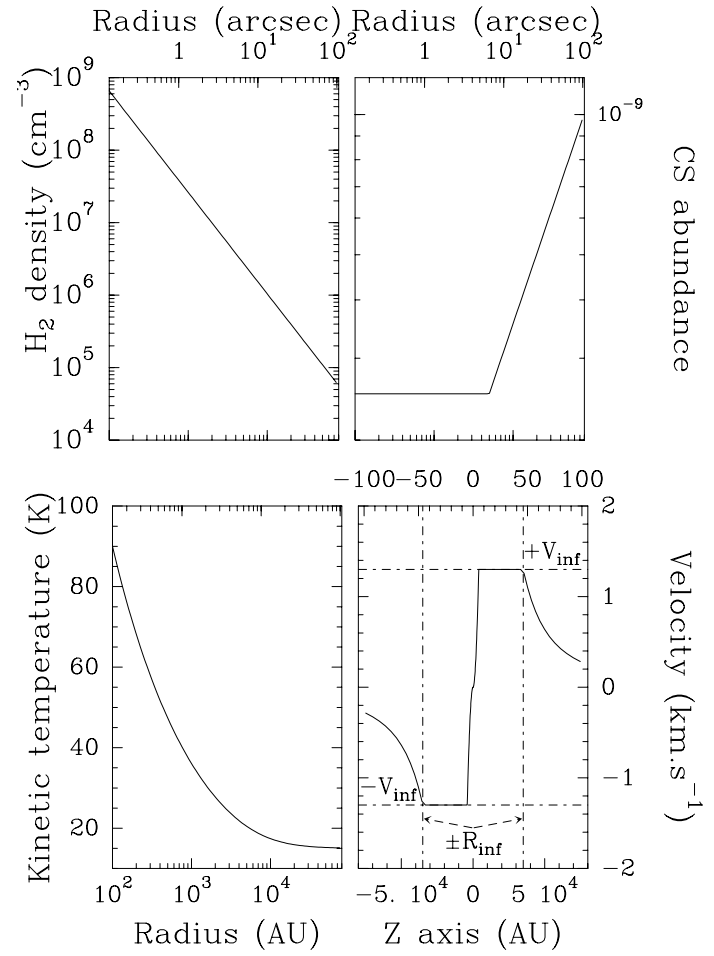

Fig. 9. Profiles of the input parameters adopted in our 2D radiative transfer model of NGC 2264-C: Density scaling as $n \propto r^{-1.4}$; depletion of the CS abundance by a factor of $\sim 5$ in the inner region (the $\mathrm{C}^{34} \mathrm{~S}$, $\mathrm{H}^{13} \mathrm{CO}^{+}$, and $\mathrm{HCO}^{+}$abundances are assumed to scale accordingly); gas kinetic temperature scaling as $T_{k} \propto r^{-0.4}$ at small radii and fixed to $T_{k}=15 \mathrm{~K}$ in the outer region; velocity profile along the $z$-axis of the cylinder (solid lines), with the locations of $\pm V_{\text {inf }}$ and $\pm R_{\text {inf }}$ marked by dash-dotted lines.

Figure 10 compares the synthetic spectra calculated under these assumptions with the spectra observed along the EW axis in the $\mathrm{CS}(3-2), \mathrm{CS}(5-4), \mathrm{C}^{34} \mathrm{~S}(2-1), \mathrm{C}^{34} \mathrm{~S}(3-2), \mathrm{C}^{34} \mathrm{~S}(5-4)$, $\mathrm{HCO}^{+}(3-2), \mathrm{H}^{13} \mathrm{CO}^{+}(3-2)$, and $\mathrm{H}^{13} \mathrm{CO}^{+}(1-0)$ transitions (where the $\left(0^{\prime \prime}, 0^{\prime \prime}\right)$ position corresponds to C-MM3). This agreement between the simulations and the observations is very encouraging given the simplicity of the model. The linewidths, peaks, and dips of the observed spectra are generally reproduced well. Furthermore, our simple cylindrical model manages to reproduce the observed reversal in line asymmetry, from blue-skewed spectra near C-MM3 and on the Eastern side of C-MM3 to red-skewed spectra on the Western side of C-MM3 (cf. Fig. 10), without including any additional outflow velocity component near C-MM4. On the Western side of C-MM3, however, the intensity of the synthetic spectra is not strong enough, especially in the case of the optically thick $\mathrm{HCO}^{+}(3-2)$ and $\mathrm{CS}(5-4)$ transitions. This is because our $1 \mathrm{D}$ model underestimates both the actual density and the actual kinetic temperature, hence the excitation temperature, near the luminous IR source IRS1 and the millimeter sources C-MM4 and C-MM5, which are all offset from the center of gravity of the system at C-MM3.

We note that both the observed and synthetic $\mathrm{H}^{13} \mathrm{CO}^{+}(1-0)$ spectra are asymmetric and even double-peaked near C-MM3. This is somewhat reminiscent of the asymmetry observed in
$\mathrm{HCO}^{+}(3-2)$, although there is a fundamental difference. While the $\mathrm{HCO}^{+}(3-2)$ spectra are optically thick and self-absorbed (with an optical depth $\tau \sim 45$ in the central velocity channel of the model), the $\mathrm{H}^{13} \mathrm{CO}^{+}(1-0)$ transition is essentially optically thin (with $\tau \sim 0.6$ in the central velocity channel of the model), so that the asymmetric, double-peaked $\mathrm{H}^{13} \mathrm{CO}^{+}(1-0)$ profiles reflect the presence of two velocity components along the line-of-sight (see also the position-velocity diagrams shown in Fig. 6) rather than self-absorption effects.

Figure 11 provides a schematic explanation of the line reversal for optically thick spectra. Three lines of sight (1.o.s. 1, 1.o.s. 2, and 1.o.s. 3) are represented, which intersect different isocontours of excitation temperature. Due to the elongated, cylindrical structure of the model, the blueshifted material probed along l.o.s. 3 has much lower excitation and/or column density than the blueshifted material along 1.o.s. 2 or 1.o.s. 1 . This explains why the intensity of the blue peak of the selfabsorbed lines is strong on the Eastern side of C-MM3 and decreases rapidly on the Western side (see Fig. 10). By contrast, the two lines of sight, 1.o.s. 2 and l.o.s. 3, include similar amounts of excited, redshifted material, which qualitatively explains why the red peak remains approximately constant (in the model) on the Western side of C-MM3. Of course, protostellar outflows are likely to play an additional role in shaping the line profiles observed in this region, especially around C-MM4 and IRS 1; see the broad wings of the central CS(3-2) spectrum observed toward C-MM4 in Fig. 10. Nevertheless, the qualitative explanation above of the asymmetry reversal and the reasonably good model fit shown in Fig. 10 suggest that the velocity field near C-MM3 is dominated by the global collapse of the entire NGC 2264-C elongated clump rather than by local inflowing/outflowing motions around individual sources.

In order to estimate rough error bars on our "best-fit" model shown in Fig. 10, we performed a set of radiative transfer calculations by varying some of the model parameters. We found acceptable fits for an inflow velocity $V_{\text {inf }}=1.3 \mathrm{~km} \mathrm{~s}^{-1} \pm$ $0.2 \mathrm{~km} \mathrm{~s}^{-1}$ and an inflow radius $R_{\text {inf }}=35000 \mathrm{AU} \pm 10000 \mathrm{AU}$; see Fig. 9 for definitions of these two parameters. As an illustration, Fig. 12 shows synthetic $\mathrm{H}^{13} \mathrm{CO}^{+}(1-0)$ positionvelocity diagrams for three different models: (a) the "bestfit" model; (b) a model similar to the best-fit model but with $R_{\text {inf }}=20000 \mathrm{AU}$ instead of $R_{\text {inf }}=35000 \mathrm{AU}$; and (c) a model for which the velocity field corresponds to (differential) rotation about C-MM3, as opposed to inflow toward C-MM3. It can be seen that the agreement between the best-fit PV diagram (Fig. 12a) and the observed diagram (top right of Fig. 6) is quite good, with two separate velocity components overlapping at the central position. By contrast, the model with a smaller inflow radius (Fig. 12b) does not match the main features of the observed PV diagram, even if an unresolved velocity discontinuity is apparent in the center. Likewise, the rotation model does not fit the observed PV diagram, producing a continuous velocity gradient as opposed to a velocity discontinuity. Based on the rotational models we calculated, we can rule out rotation as the origin of the kinematic properties of NGC 2264-C. We also tried but failed to reproduce our observations with the kinematical model proposed by Williams \& Garland (2002), namely large-scale spherical infall onto an 

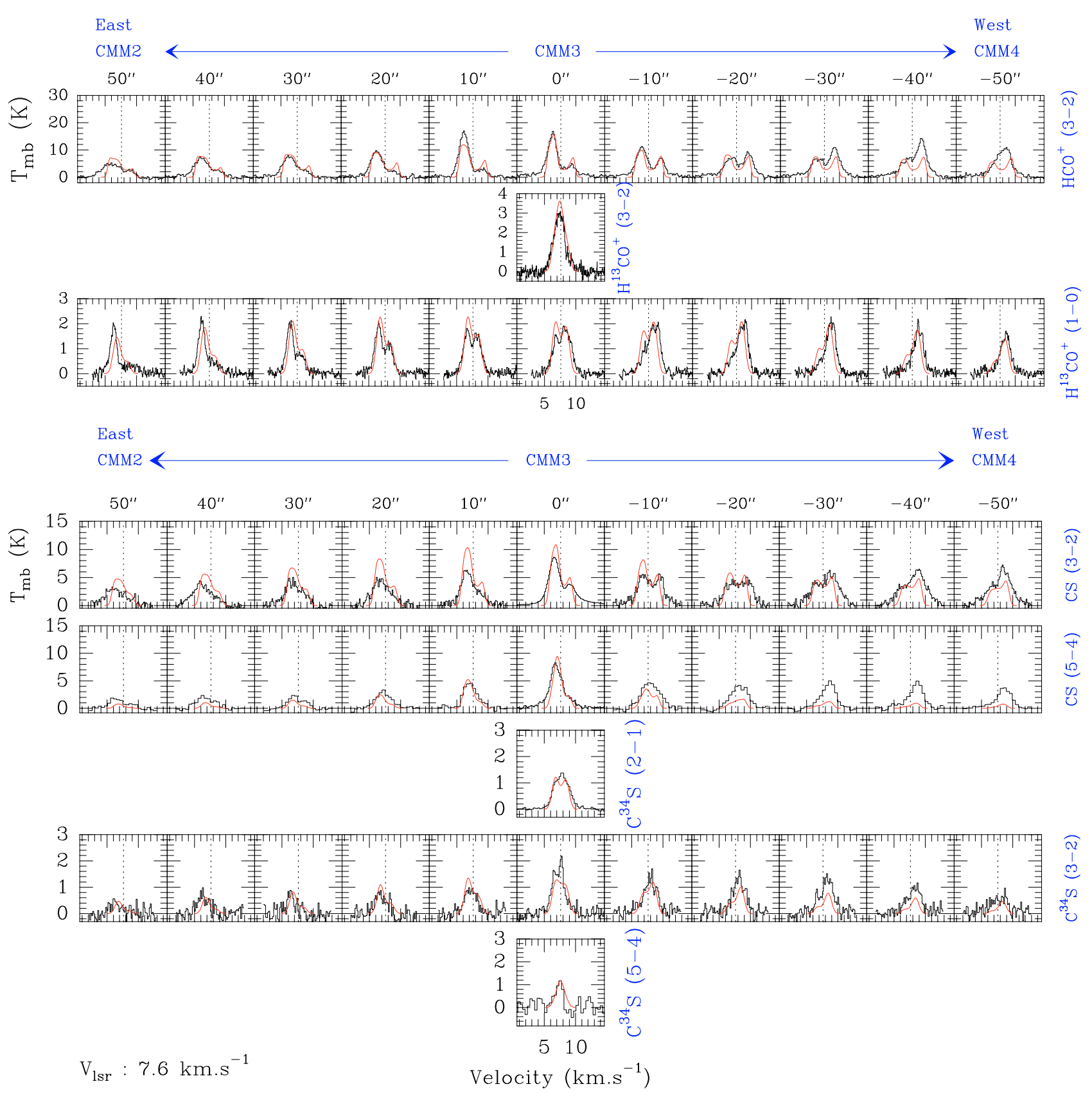

Fig. 10. $\mathrm{HCO}^{+}(3-2), \mathrm{H}^{13} \mathrm{CO}^{+}(3-2), \mathrm{H}^{13} \mathrm{CO}^{+}(1-0)$ (top) and $\mathrm{CS}(3-2), \mathrm{CS}(5-4), \mathrm{C}^{34} \mathrm{~S}(2-1), \mathrm{C}^{34} \mathrm{~S}(3-2), \mathrm{C}^{34} \mathrm{~S}(5-4)$ (bottom) spectra observed along an East-West axis going through C-MM2, C-MM3, C-MM4 in NGC 2264-C. Synthetic spectra corresponding to the "best-fit" 2D radiative transfer model described in the text (see Fig. 8c for input parameters) are superimposed in light grey. The central position corresponds to $\mathrm{C}-\mathrm{MM} 3$.

expanding central core coinciding with C-MM4. Our higherresolution observations show that the large-scale collapse motions in NGC 2264-C converge toward a position much closer to C-MM3 than to C-MM4 and that these motions are not disrupted on small scales by the effect of protostellar outflows around C-MM4 as proposed by Williams \& Garland (2002).

Based on our best-fit model (cf. Fig. 10), the dynamical timescale of the inner part of NGC 2264-C is estimated to be $t_{\mathrm{dyn}} \sim 1.7 \times 10^{5} \mathrm{yr}$, which corresponds to the characteristic time needed by C-MM2 and C-MM4 to collapse to the system's central position (C-MM3). We can also calculate the mass inflow rate toward the center of the cylindrical filament $\dot{M}_{\text {inf }}=2 \times \pi R_{\text {fil }}^{2} \times n_{\text {mean }} \times \mu \times m \times V_{\text {inf }}$, where $R_{\text {fil }}$ is the radius of the cylinder cross section, $n_{\text {mean }}$ the mean number density in the cylinder, $\mu$ the mean molecular weight, $m$ the mass of atomic hydrogen, and $V_{\text {inf }}$ the inflow velocity used in our model fit. With $R_{\text {fil }}=0.2 \mathrm{pc}, n_{\text {mean }}=1 \times 10^{5} \mathrm{~cm}^{-3}$ (cf. Table 6), $\mu=2.33$, and $V_{\text {inf }}=1.3 \mathrm{~km} \mathrm{~s}^{-1}$, we find $\dot{M}_{\text {inf }} \sim 3 \times 10^{-3} M_{\odot} \mathrm{yr}^{-1}$. This is an order of magnitude larger than the mass inflow rate found by Williams \& Garland (2002), which is not surprising since, in our picture, their spherical model was offset by $\sim 0.15 \mathrm{pc}$ from the true center of mass of NGC 2264-C. 


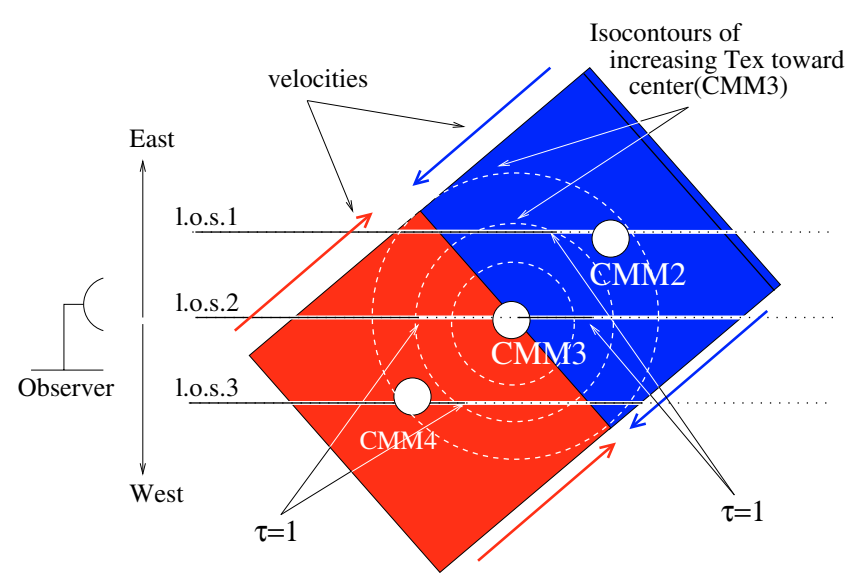

Fig. 11. Schematic view of the collapsing NGC 2264-C filament explaining the reversal of self-absorbed asymmetry observed in optically thick transitions (see Sect. 6). The positions of C-MM2, C-MM3, and C-MM4 are marked. The white dashed circles represent isocontours of increasing excitation temperature toward C-MM3. Three lines of sight (l.o.s.) are shown and the point where the optical depth $\tau=1$ along each of them is indicated.

\section{Radiative transfer modelling of D-MM1}

As the mean separation between nearest MMSs is larger in NGC 2264-D than in NGC 2264-C, our molecular linemapping observations of clump D should be more sensitive to the dynamics of individual MMSs. In this section, we present an attempt at modelling the strongest millimeter source of NGC 2264-D, D-MM1, which was classified as a Class 0 protostar by Wolf-Chase et al. (2003) and which exhibits blue infall profiles in both $\mathrm{HCO}^{+}(3-2)$ and $\mathrm{CS}(3-2)$ (see, e.g., Fig. 7).

We modelled D-MM1 as a spherical cloud core with both infall and rotational motions using the same radiative transfer code (MAPYSO) as for NGC 2264-C (Sect. 6). Several input parameters of the code are well-constrained. We assumed the gas kinetic temperature to be coupled well to the dust temperature and adopted a dust temperature profile $T_{\mathrm{d}} \propto r^{-0.4}$ with $T_{\mathrm{d}} \sim 80 \mathrm{~K}$ at $r=100 \mathrm{AU}$ (cf. Motte \& André 2001 and references therein), consistent with the observed bolometric luminosity $L_{\text {bol }} \sim 100 L_{\odot}$ (Wolf-Chase et al. 2003). From our $1.2 \mathrm{~mm}$ continuum map, we derived a circularly-averaged radial flux density profile that is consistent with a radial density profile $\rho \propto r^{-1.5}$ in the inner $1 \times 10^{4} \mathrm{AU}$ radius region and steeper outside with $\rho \propto r^{-2}$. The model density profile was normalized such that the mass of the cloud core was $10 M_{\odot}$ within a radius of $4500 \mathrm{AU}$ as observed. The non-thermal velocity dispersion was set to $\sigma_{\mathrm{NT}}=0.51 \mathrm{~km} \mathrm{~s}^{-1}$ which is slightly less than the observed linewidth (cf. Table 2), due to the collapse contribution to the linewidth. The $\mathrm{N}_{2} \mathrm{H}^{+}(1-0)$ spectra we observed around D-MM1 indicated the presence of a relatively strong velocity gradient $\sim 3 \mathrm{~km} \mathrm{~s}^{-1} \mathrm{pc}^{-1}$ from south-west to north-east $\left(\mathrm{PA}_{\text {grad }} \sim+45^{\circ}\right)$. In agreement with our $\mathrm{N}_{2} \mathrm{H}^{+}$ data, the projection of the rotation axis on the plane of the sky was thus fixed at $\mathrm{PA}_{\text {rot }} \sim-45^{\circ}$, and an angular velocity $\Omega=3 \mathrm{~km} \mathrm{~s}^{-1} \mathrm{pc}^{-1}$ was adopted at $r=2 \times 10^{4}$ AU from the center of D-MM1.
Synthetic $\mathrm{H}^{13} \mathrm{CO}^{+}(1-0)$ position-velocity diagrams
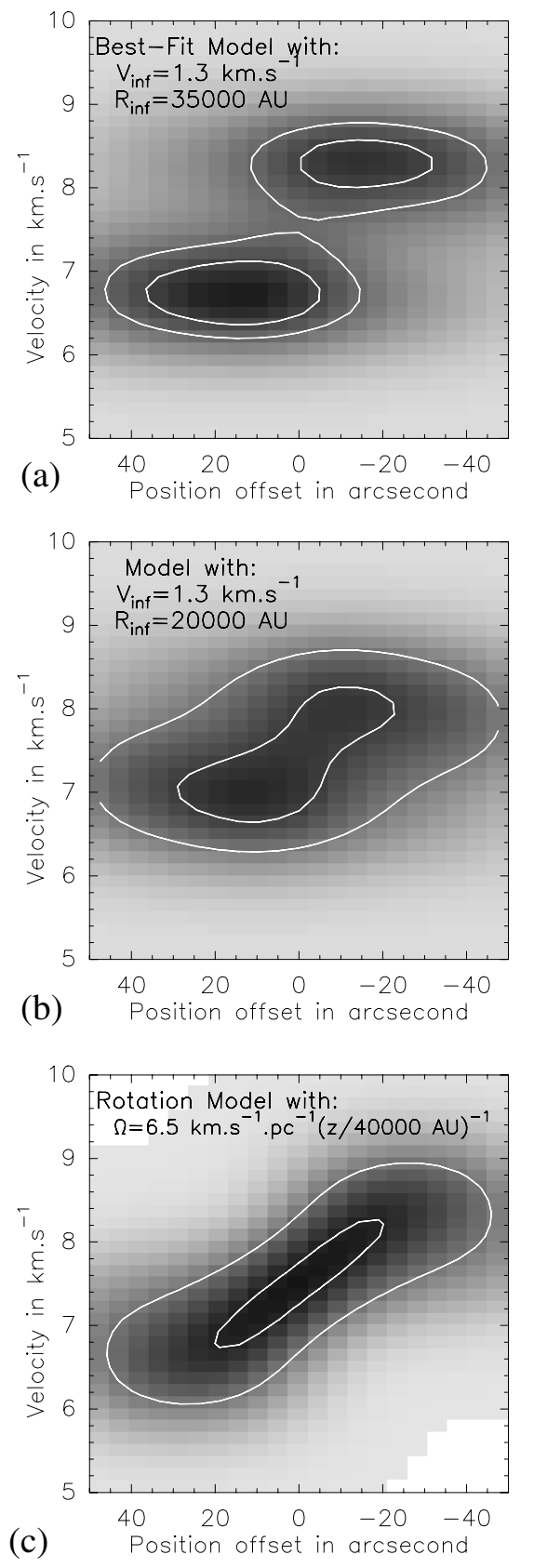

Fig. 12. Synthetic $\mathrm{H}^{13} \mathrm{CO}^{+}(1-0)$ position-velocity diagrams calculated for three different radiative transfer models. a) Diagram corresponding to the "best-fit" model (see text for details). b) Diagram obtained by changing $R_{\text {inf }}$ to 20000 AU. c) Diagram obtained for a bulk (differential) rotation of the model cloud about an axis centered on C-MM3 and perpendicular to the line joining C-MM2, C-MM3, and C-MM4. These diagrams have to be compared with the observed $\mathrm{H}^{13} \mathrm{CO}^{+}(1-0)$ diagram shown at the top right of Fig. 6 .

Taking the above constraints into account, the magnitudes of the infall and rotational velocities were adjusted so as to match the observations. In the model shown in Figs. 13 and 14, the infall velocity is such as $V_{\text {inf }} \propto r^{-0.5}$ and fixed to a value of $0.2 \mathrm{~km} \mathrm{~s}^{-1}$ at $r=4000 \mathrm{AU}$. In order to reproduce the spatial sequence of self-absorbed $\mathrm{HCO}^{+}$and $\mathrm{CS}$ spectra (clearly 

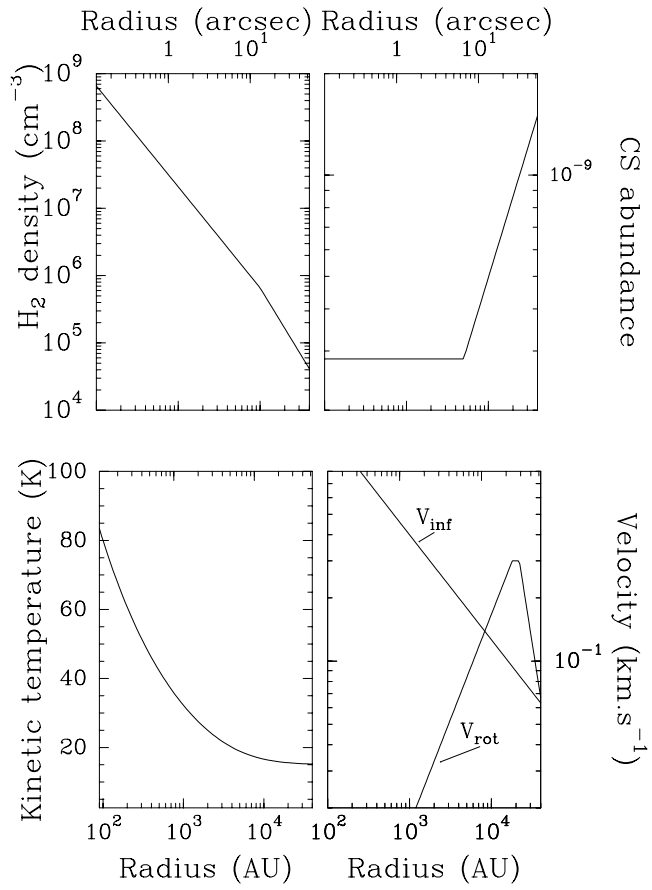

Fig. 13. Radial profiles of the input parameters adopted in our 2D radiative transfer model of D-MM1: Density scaling as $n \propto r^{-1.5}$ in the inner $10000 \mathrm{AU}$ and $n \propto r^{-2}$ beyond $10000 \mathrm{AU}$; depletion of the CS abundance by a factor of $\sim 6$ in the inner region (the profiles of the $\mathrm{C}^{34} \mathrm{~S}, \mathrm{HCO}^{+}$, and $\mathrm{H}^{13} \mathrm{CO}^{+}$are assumed to have the same form); $\mathrm{ki}$ netic temperature scaling as $T_{k} \propto r^{-0.4}$; infall velocity profile (marked $V_{\text {inf }}$ ) and rotational velocity profile (marked $V_{\text {rot }}$ and depending only on the cylindrical radius from the rotation axis).

skewed to the blue at negative offsets but almost symmetric at positive offsets - see Fig. 14), we found that strong differential rotation was required, with, e.g., solid-body rotation, $V_{\text {rot }} \propto r$, in the inner $2 \times 10^{4} \mathrm{AU}$ radius region and a sharp decline, $V_{\text {rot }} \propto r^{-2.5}$, in the outer region. Such a rotational velocity profile is reminiscent of the velocity field found by Belloche et al. (2002) for the low-mass Class 0 object IRAM 04191 in Taurus. With these parameters and assumptions (summarized in Fig. 13), a reasonably good fit was found (see Fig. 14), although the model fails to reproduce the broad wings observed in $\mathrm{HCO}^{+}(3-2)$ and $\mathrm{CS}(3-2)$, probably due to outflowing material.

Based on this model fit, we estimated the mass infall rate onto D-MM1 as follows: $\dot{M}_{\mathrm{DMM} 1}=4 \pi R^{2} \times n_{\mathrm{H}_{2}} \times \mu \times m \times V_{\text {inf }}$. At a radius of $10000 \mathrm{AU}, V_{\mathrm{inf}} \simeq 0.1 \mathrm{~km} \mathrm{~s}^{-1}$ and $n_{\mathrm{H}_{2}} \sim 6 \times 10^{5} \mathrm{~cm}^{-3}$, which yields $\dot{M}_{\text {DMM1 }}=1.1 \times 10^{-4} M_{\odot} \mathrm{yr}^{-1}$. This is almost two orders of magnitude higher than the typical infall rate measured toward low-mass protostars in Taurus (Ohashi 1999; Belloche et al. 2002), and comparable to the large infall rate estimated for some Class 0 protostars in protoclusters such as NGC 1333-IRAS4A (cf. Di Francesco et al. 2001; André et al. 2004). The implications of such a high infall rate are discussed in the next section.
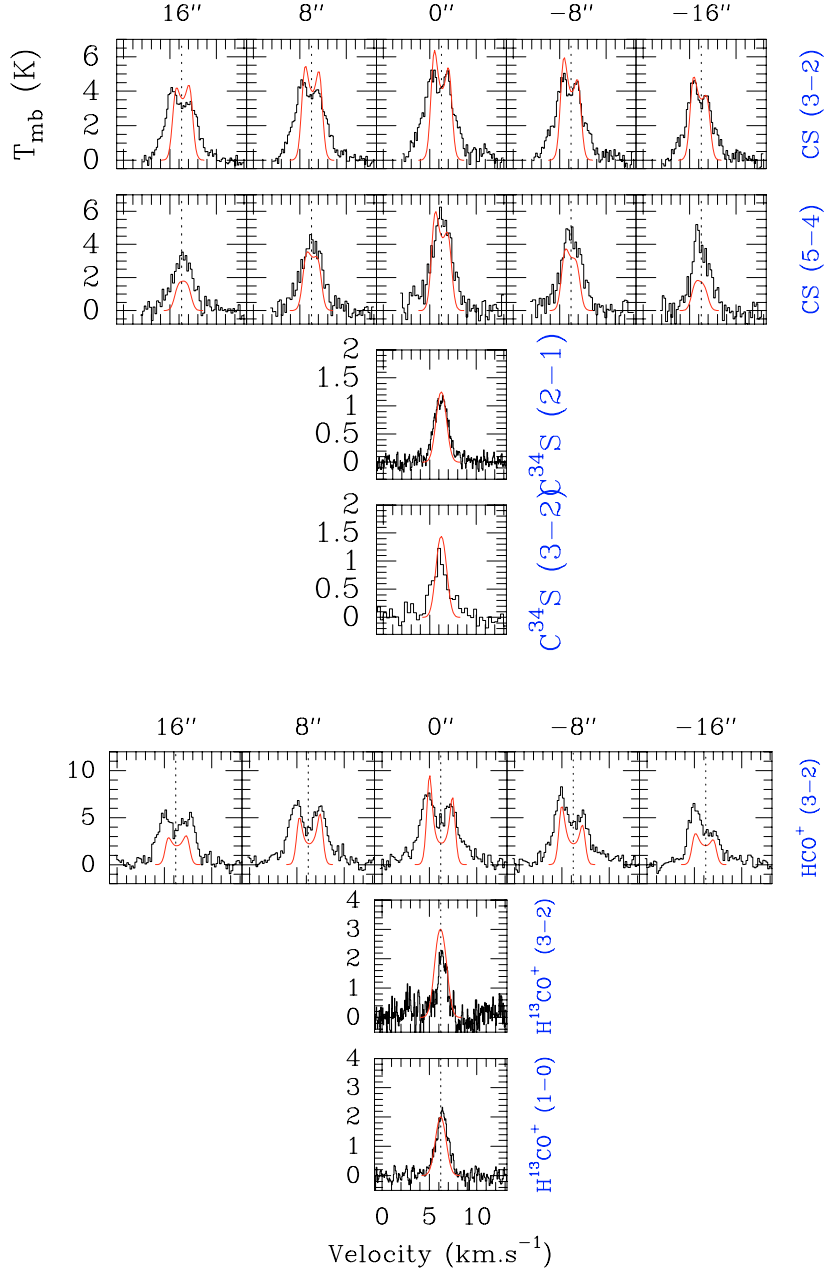

Fig. 14. $C S(3-2), \quad C S(5-4), \quad C^{34} S(2-1), \quad C^{34} S(3-2) \quad$ (top), and $\mathrm{HCO}^{+}(3-2), \mathrm{H}^{13} \mathrm{CO}^{+}(3-2), \mathrm{H}^{13} \mathrm{CO}^{+}(1-0)$ (bottom) spectra observed along the direction of maximum velocity gradient $\left(\mathrm{PA}_{\text {grad }} \sim+45^{\circ}\right)$ through D-MM1. Synthetic spectra corresponding to the "best-fit" $2 \mathrm{D}$ radiative transfer model described in the text are superimposed in light grey. Positive offsets are located to the North-East of D-MM1, while negative offsets are to the South-West.

\section{Discussion and conclusions}

\subsection{Comparison of three protoclusters: NGC 2264-C, NGC 2264-D, and $\rho$-Ophiuchi}

The overall dynamical state and stability of a clump may influence the formation process of individual stars in its interior. We have shown in Sect. 6 that the NGC 2264-C clump is in a state of global collapse and evolves on the dynamical timescale $t_{\text {dyn }} \sim 1.7 \times 10^{5}$ yr. This implies that NGC $2264-C$ is not in approximate hydrostatic equilibrium. Independently, we can compare the $3 \mathrm{D}$ velocity dispersion of the MMSs, $\sigma_{3 \mathrm{D}}$, to the $3 \mathrm{D}$ velocity dispersion expected in virial equilibrium, $\sigma_{\mathrm{vir}} 3 \mathrm{D}$. These parameters are given in Table 6 for both NGC 2264-C and NGC 2264-D, as well as $\rho$-Ophiuchi, the nearest $(d \sim$ $150 \mathrm{pc}$ ) example of a cluster-forming clump. Table 6 also lists the free-fall time of each clump estimated from the observed mean volume density. In all three cases, the observed velocity dispersion of the MMSs appears to be insufficient to balance 
Table 6. Global properties of NGC 2264-C and NGC 2264-D compared to those of $\rho$-Oph.

\begin{tabular}{ccccccccc}
\hline \hline Cl. name & $\begin{array}{c}\text { Cl. diam. }^{a} \\
(\mathrm{pc})\end{array}$ & $\begin{array}{c}\text { Cl. mass }^{b} \\
\left(M_{\odot}\right)\end{array}$ & $\begin{array}{c}M_{\text {vir }}{ }^{c} \\
\left(M_{\odot}\right)\end{array}$ & $\begin{array}{c}\text { Cl. col. dens. } \\
\left(10^{22} \mathrm{~cm}^{-2}\right)\end{array}$ & $\begin{array}{c}\text { Cl. vol. dens. }{ }^{e} \\
\left(10^{4} \mathrm{~cm}^{-3}\right)\end{array}$ & $\begin{array}{c}t_{\mathrm{ff}}{ }^{f} \\
\left(10^{5} \mathrm{yr}\right)\end{array}$ & $\begin{array}{c}\sigma_{\text {3D }^{g}} \\
\left(\mathrm{~km} \mathrm{~s}^{-1}\right)\end{array}$ & $\begin{array}{c}\sigma_{\text {vir_3D }^{h}} \\
\left(\mathrm{~km} \mathrm{~s}^{-1}\right)\end{array}$ \\
\hline NGC2264-C & 0.8 & 1650 & 340 & 16 & 10.7 & 1.0 & 1.3 & 3.7 \\
NGC2264-D & 0.9 & 1310 & 310 & 11 & 6.0 & 1.4 & 1.4 & 3.0 \\
\hline$\rho-O$ Oph $^{i}$ & 1.1 & 550 & - & 3 & 1.4 & 2.9 & 0.6 & 2.1 \\
\hline
\end{tabular}

${ }^{a}$ Mean clump diameter measured at the $70 \mathrm{mJy} / 11^{\prime \prime}$-beam contour in the $1.2 \mathrm{~mm}$ continuum map (cf. Figs. $3 \mathrm{a}$ and $3 \mathrm{~b}$ ).

${ }^{b}$ Total gas mass of the clump estimated from the $1.2 \mathrm{~mm}$ continuum image prior to spatial filtering.

$c$ Virial mass calculated in the same way as in Table 2 from the mean $\mathrm{N}_{2} \mathrm{H}^{+}(1-0)$ line-of-sight velocity dispersion observed in the clump (cf. Col. 3 of Table 3).

${ }^{d}$ Mean column density averaged over each clump.

$e$ Mean density of each clump assuming a spherical volume.

${ }^{f}$ Mean free-fall time of the clump.

${ }^{g}$ Observed 3D velocity dispersion of the MMSs in each clump.

${ }^{h}$ Expected 3D velocity dispersion of the MMSs if they were virialized in the gravitational potential of the clump: $\sigma_{\text {vir_3D }}=\sqrt{\frac{G M_{1.2}}{R}}$.

${ }^{i}$ Based on the $\mathrm{C}^{18} \mathrm{O}(1-0)$ results of Wilking \& Lada (1983) for the diameter and the mass, and on Belloche et al. (2001) for the velocity dispersion.

gravity. Likewise, the estimated virial mass $M_{\mathrm{vir}}$ is significantly smaller than the total gas mass derived from the $1.2 \mathrm{~mm}$ continuum map for each clump (cf. Table 6), corresponding to a virial parameter $\alpha_{\mathrm{vir}}=M_{\mathrm{vir}} / M_{1.2} \simeq 0.2$, which suggests that both NGC 2264-C and NGC 2264-D are out of virial equilibrium and very unstable to collapse. If the clumps are gravitationally unstable, we expect the free-fall time to provide a reasonably good estimate of the characteristic evolutionary timescale. Our independent estimate of $t_{\mathrm{dyn}}$ for NGC 2264-C (see above) supports this view, since $t_{\text {dyn }}$ is only slightly larger than $t_{\mathrm{ff}}$. We conclude that the central part of NGC 2264-C is close to being in free-fall collapse. NGC 2264-D and $\rho$-Ophiuchi may also be collapsing. Using $\mathrm{HCO}^{+}(3-2)$, Williams \& Garland (2002) found some evidence of large-scale collapse in NGC 2264-D at a characteristic velocity $v_{\text {coll }}=0.3 \mathrm{~km} \mathrm{~s}^{-1}$, although more observations of optically-thick tracers on large scales would be required to confirm their results. Likewise, tentative signatures of large-scale infall have been observed in ${ }^{12} \mathrm{CO}$ and ${ }^{13} \mathrm{CO}$ toward the $\rho$ Oph clump (e.g. Encrenaz et al. 1975; Wilking \& Lada 1983).

We then compared the MMSs of NGC 2264 with the $\mathrm{DCO}^{+}$cores of $\rho$ Oph (Loren et al. 1990) and typical isolated prestellar cores (Ward-Thompson et al. 1999; Caselli et al. 2002). Figure 15 shows a plot of mean pressure, $\bar{P}_{\text {core }} / k$, versus non-thermal velocity dispersion, $\sigma_{\mathrm{NT}}$, for these various types of cores, as well as a typical singular isothermal sphere (SIS) model (Shu et al. 1987) and a typical turbulent core model (McKee \& Tan 2003 - hereafter MT03). The mean pressure in each core was calculated from its mean column density derived from millimeter continuum observations, using Eqs. (A5)-(A6) of MT03 and assuming $\alpha_{\text {vir }}=1$, which is typical of the NGC 2264 MMSs (see Table 2). The non-thermal component of the $1 \mathrm{D}$ velocity dispersion in each core was estimated from the observed $\mathrm{N}_{2} \mathrm{H}^{+}(101-012)$ linewidth after subtracting the thermal broadening expected for $T_{k}=10 \mathrm{~K}$.

Figure 15 shows that $\bar{P}_{\text {core }} / k$ increases by three orders of magnitude and that $\sigma_{\mathrm{NT}}$ increases by one order of magnitude from the thermally-dominated, isolated prestellar cores (cf. Myers 1998) to the most extreme MMSs of NGC 2264 (this paper) dominated by supersonic non-thermal motions. It can also be noted that the hydrostatic SIS model provides a reasonably good representation of the locations of isolated prestellar cores in the $\bar{P}_{\text {core }} / k$ vs. $\sigma_{\mathrm{NT}}$ diagram, while the turbulent core model of MT03 (see Sect. 8.2 below) provides a much better description of the NGC 2264 sources. Interestingly, the low-pressure, thermally-dominated cores on the left-hand side of Fig. 15 typically form one (or two) stars, while the highpressure, "turbulent" cores on the right-hand side are associated with protoclusters. A similar distinction between the "isolated" and "clustered" modes of star formation was noted earlier by Myers (1998) and Jijina et al. (1999).

In the high-pressure, high- $\sigma_{\mathrm{NT}}$ part of Fig. 15, it can be seen that the $\rho$-Oph cores Oph A and Oph B2 are located in the regime of the MMSs of NGC 2264-D. The sources of NGC 2264-C have higher mean pressure and larger nonthermal velocity dispersions by a factor $\sim 10$ and $\sim 2$ on average, respectively, than Oph A and Oph B2. The most extreme source of NGC 2264-C (C-MM3) has a mean pressure $\bar{P}_{\text {core }} / k \sim 10^{10} \mathrm{~K} \mathrm{~cm}^{-3}$ reminiscent of the pressure found in high-mass star-forming clumps (cf. MT03).

We conclude that the MMSs of NGC 2264, especially those of clump C, represent somewhat more pressurized and more turbulent conditions for star formation than do the $\rho$ Oph cores. The more extreme conditions found in NGC 2264-C are actually reminiscent of those suggested by MT03 for the precursors of massive stars.

\subsection{Comparison with two scenarios of clustered star formation}

The young near-infrared star cluster associated with NGC 2264 (Lada et al. 1993) is known to be highly structured, and it exhibits a surface density distribution with multiple peaks (see 


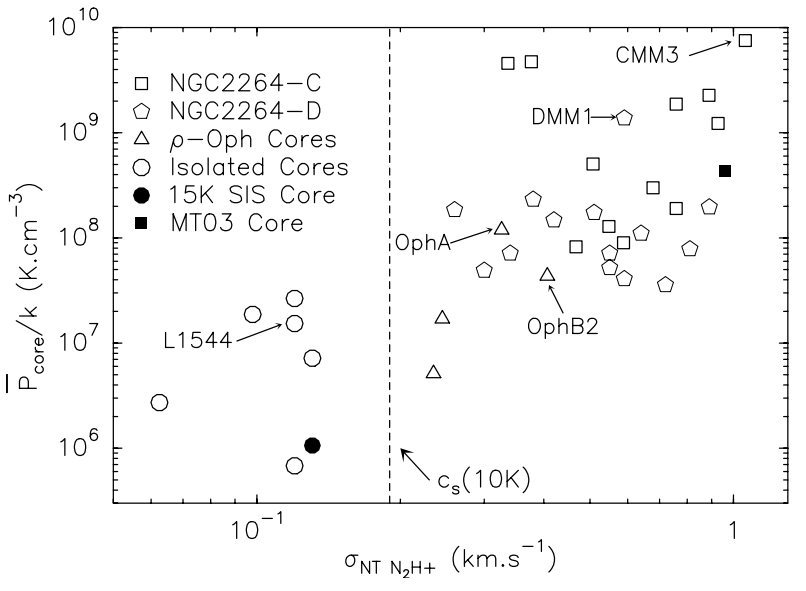

Fig. 15. Plot of mean pressure versus non-thermal velocity dispersion for the millimeter continuum sources identified in NGC 2264-C (square symbols) and NGC 2264-D (pentagonal symbols), the $\rho$ Oph $\mathrm{DCO}^{+}$cores Oph A, OphB 2, Oph C, Oph F (open triangles - from MAN98 and Belloche et al. 2001), and the isolated prestellar cores L1512, L1544, L183, L1696A, L63, L1155C (open circles - from Ward-Thompson et al. 1999 and Caselli et al. 2002). The location of a singular isothermal sphere (SIS) model with an effective temperature of $15 \mathrm{~K}$ and an actual gas temperature of $10 \mathrm{~K}$ is shown as a filled circle for comparison; its mean pressure was estimated within a radius $R=0.035 \mathrm{pc}$, typical of isolated prestellar cores (e.g. Ward-Thompson et al. 1999). The filled square corresponds to the turbulent core model of McKee \& Tan (2003 - MT03) for a clump surface density $\Sigma_{\mathrm{cl}}=1 \mathrm{~g} \mathrm{~cm}^{-2}$ and a final stellar mass $m_{\star f}=10 M_{\odot}$ (see also Sect. 8.2). The vertical dashed line separates cores dominated by thermal motions (on the left) from cores dominated by supersonic non-thermal motions (on the right), assuming a typical gas temperature of $10 \mathrm{~K}$.

Fig. 4 of Lada \& Lada 2003). Two of these peaks lie close to, but are clearly offset from, the cluster-forming clumps NGC 2264-C and NGC 2264-D studied in the present paper (see Fig. 16). This is suggestive of at least two different episodes of (clustered) star formation in the NGC 2264 region. Detection of a few 2MASS and MSX infrared sources toward both NGC 2264-C and NGC 2264-D shows that both clumps have already formed YSOs. Furthermore, the presence of the massive (B2-type) Class I infrared YSO IRS1 within the NGC 2264-C clump sets interesting constraints on the process of intermediate- to high-mass star formation.

According to the scenario proposed by Bonnell et al. (1998, 2002), formation of massive stars occurs through competitive accretion and stellar mergers in the dense inner core of contracting protoclusters when their central stellar density exceeds $\sim 10^{8}$ stars $/ \mathrm{pc}^{3}$. The gravitational potential well of such cluster cores is dominated by the stellar rather than the gas component. We find that it is difficult to account for the formation of IRS1 in the context of this scenario. Indeed, IRS1 appears to be located in the outer parts of the NGC 2264-C gas clump (e.g. Thompson et al. 1998; Nakano et al. 2002), rather near the peak of the near-IR source density distribution (cf. Fig. 16). Figure 1 in Lada et al. (1993) indicates a stellar surface density $\sim 80 \mathrm{pc}^{-2}$, which allows us to estimate the stellar contribution to the gravitational potential within the volume of NGC 2264-C. Assuming the Kroupa (2001) IMF, for which the

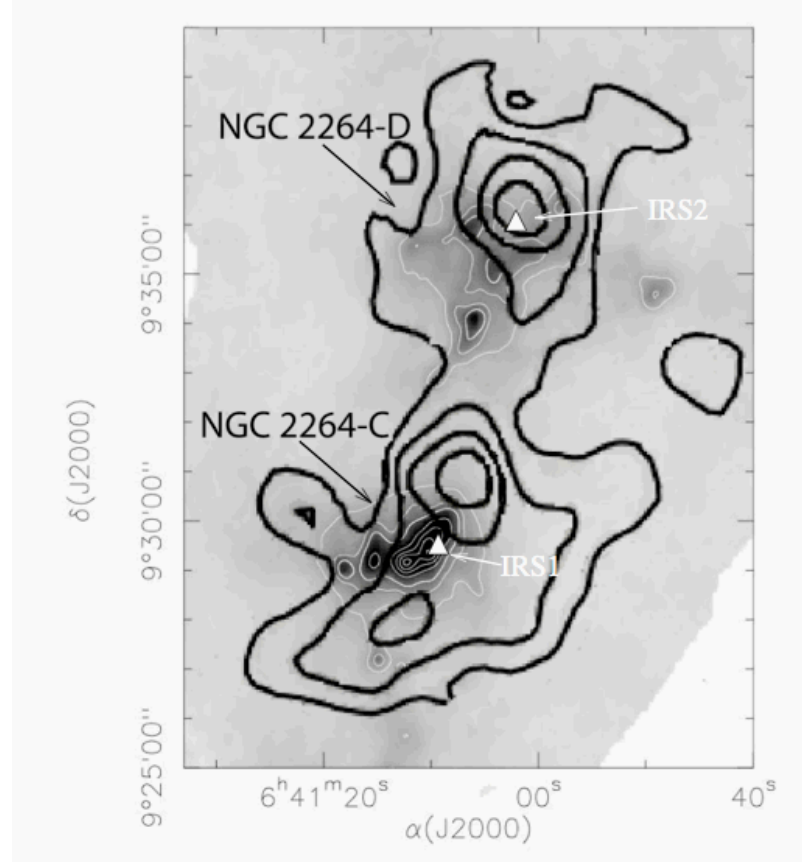

Fig. 16. Contour map of the surface density of near-IR sources in the young star cluster associated with NGC 2264 (from Lada \& Lada 2003), overlaid on our $1.2 \mathrm{~mm}$ continuum mosaic of the region (greyscale and white contours). The white filled triangles mark the positions of IRS1 and IRS2. Note that the maxima of the nearIR source surface density distribution are located outside the dense gas/dust clumps NGC 2264-C and NGC 2264-D, while the projected position of the luminous near-IR source IRS1 lies within the $1.2 \mathrm{~mm}$ contours of clump C

mean stellar mass is $\sim 0.4 M_{\odot}$, the above number surface density translates into a stellar mass surface density $\sim 30 M_{\odot} \mathrm{pc}^{-2}$, i.e., less than $2 \%$ of the total gas mass surface density. Even allowing for a possible fraction of highly extinguished stars in the protocluster that would not be included in the above estimate, we conclude that the gravitational potential of NGC 2264-C is dominated by the gas rather than by the stars. Thus, IRS1, the most massive infrared YSO in this region is unlikely to have formed by collision and coalescence of lower-mass stars as described by Bonnell et al. (1998, 2002). On the other hand, the global, near free-fall collapse of NGC 2264-C may lead to strong dynamical interactions between MMSs and even protostellar mergers near the center of the gas-dominated clump (see below).

In the alternative scenario proposed by McKee \& Tan (2002, 2003 - e.g. MT03), high-mass stars form from massive, turbulent cores embedded in a high-pressure, environment/clump. Both the cores and the ambient medium are assumed to be centrally-concentrated and initially virialized, i.e., close to hydrostatic equilibrium. Although clumps C and D are indeed massive and turbulent, our observations reveal that they are not in hydrostatic equilibrium, but rather in a state of global dynamical collapse, at least in the case of NGC 2264-C. The primary characteristic of the MT03 model, however, is that the prestellar precursors of massive protostars are high-density, 
turbulent cores, which leads to large, accelerating accretion rates during the protostellar phase. High $\left(\gtrsim 10^{-4} M_{\odot} \mathrm{yr}^{-1}\right)$ accretion rates are indeed necessary to overcome radiation pressure and allow high-mass $\left(M_{\star} \gtrsim 8 M_{\odot}\right)$ stars to form by accretion despite the high luminosity of the central stars (e.g. Wolfire \& Cassinelli 1987). Large-scale collapse can help to produce dynamically pressurized, high-density cores in the inner part of massive cluster-forming clumps. Even though one of the premises of the MT03 model is not verified, a detailed comparison of the model with the observed properties of NGC 2264-C and its central source, C-MM3, is therefore still warranted.

The mean column density of the NGC 2264-C clump is $N_{\mathrm{H} 2} \sim 1.6 \times 10^{23} \mathrm{~cm}^{-2}$, corresponding to a mean surface density $\Sigma_{\mathrm{cl}} \sim 0.6 \mathrm{~g} \mathrm{~cm}^{-2}$, typical of the one observed in most regions of massive star formation and consistent with the assumptions of the turbulent core model of MT03. With a mass of $40 M_{\odot}$ and a line velocity dispersion $\sigma_{\text {line }}=1.1 \mathrm{~km} \mathrm{~s}^{-1}, \mathrm{C}-\mathrm{MM} 3$ is located near the center of gravity of the collapsing NGC 2264-C clump. An upper limit to the mass accretion rate onto the protostellar object C-MM3 is provided by the total mass inflow rate $\dot{M}_{\text {inf }} \sim 3 \times 10^{-3} M_{\odot} \mathrm{yr}^{-1}$ derived in Sect. 6 for the central part of the NGC 2264-C. A lower limit can be estimated from the mass accretion rate onto the less extreme object D-MM1 in NGC 2264-D, $\dot{M}_{\text {DMM1 }} \sim 1.1 \times 10^{-4} M_{\odot} \mathrm{yr}^{-1}$ (cf. Sect. 7). The mass accretion rate onto C-MM3 is thus likely to be in the range $1.1 \times 10^{-4}<\dot{M}_{\mathrm{CMM} 3}<3 \times 10^{-3} M_{\odot} \mathrm{yr}^{-1}$, which is sufficient to overcome the radiation pressure of a $\sim 10 M_{\odot}$ star (Wolfire \& Cassinelli 1987; Jijina \& Adams 1996).

Assuming a typical core star-formation efficiency $\epsilon \sim 50 \%$, consistent with calculations of the feedback effect due to the protostellar outflow (e.g. Matzner \& McKee 2000), C-MM3, which is thought to be a $\sim 40 M_{\odot}$ core, could then be the progenitor to a $\sim 20 M_{\odot}$ star.

In the context of the turbulent core model, the formation of a $20 M_{\odot}$ star requires a velocity dispersion at the surface of the initial prestellar core $\sigma_{\mathrm{s}} \sim 1.0 \mathrm{~km} \mathrm{~s}^{-1}$, assuming the fiducial values of MT03 and a clump surface density $\Sigma_{\mathrm{cl}} \sim 0.6 \mathrm{~g} \mathrm{~cm}^{-2}$. The model then predicts a mass accretion rate $\dot{m}_{\star} \sim 2.0 \times 10^{-4} M_{\odot} \mathrm{yr}^{-1}$ when half of the final stellar mass has been accreted (i.e., end of the Class 0 phase), rising to a maximum value of $\sim 2.8 \times 10^{-4} M_{\odot} \mathrm{yr}^{-1}$ at the end of the accretion phase. In this model, the total star formation time from the birth of the protostar to the moment it reaches its final mass, $m_{\star f}=20 M_{\odot}$, is $t_{* f} \sim 1.7 \times 10^{5} \mathrm{yr}$. We conclude that the observed properties of C-MM3 in NGC 2264-C are roughly consistent with the MT03 model, albeit somewhat more extreme/dynamic than it.

The dynamical time estimated in Sect. 6, corresponding to the time for the two Class 0 protostars C-MM2 and C-MM4 to reach the position of C-MM3, is $\sim 1.7 \times 10^{5} \mathrm{yr}$. This timescale is similar to the formation time of a $20 M_{\odot}$ star in the MT03 model and to the duration of the Class I protostellar accretion phase for low-mass stars (e.g. Greene et al. 1994). Therefore, significant evolution of C-MM2 and C-MM4 is likely to occur before a merger can take place in the center of NGC 2264-C. Nevertheless, a strong dynamical interaction between C-MM2 and C-MM4 seems possible before the associated protostars have achieved their final masses. Accordingly, we suggest that the formation of an ultra-dense protostellar core through the merger of two or more intermediate-mass protostars in the central part of a protocluster may be a plausible route to high-mass star formation. Although in itself such a merger does not directly overcome the problems arising from radiation pressure, it provides a dynamical way to build up a massive protostellar core with physical characteristics similar to the McKee \& Tan model. Even if reminiscent of the Bonnell et al. $(1998,2002)$ scenario, this suggested route differs in that it would be based on the collision and coalescence of protostellar dense cores in a gas-dominated environment, as opposed to stellar mergers in a stellar-dominated potential.

\subsection{Conclusions: Massive star formation in clusters}

The picture of massive, clustered star formation that emerges from the present study is intermediate between the highly dynamic scenario of Bonnell et al. (1998) and the turbulent core scenario of MT03. In agreement with the first scenario and at variance with the second scenario, our observations support the view that protoclusters are in a state of global, dynamical collapse rather than of approximate hydrostatic equilibrium. On the other hand, in contrast to the first scenario and in better agreement with the second scenario, most stars in a cluster, including massive stars, appear to acquire their final masses while still embedded in a gas-dominated environment, with the processes occurring later on playing relatively little role.

Based on our observations, we propose a mixed scenario of protocluster formation according to which a pre-existing massive, turbulent clump is strongly compressed and induced to collapse from outside. Such a clump rapidly enters a phase of global, near free-fall collapse leading to an averaged density profile approaching $\rho \propto r^{-1.5}$ in the central regions. (Note that the presence of large-scale, supersonic inward motions may well be a generic feature of all embedded protoclusters in high-mass star-forming complexes - Motte et al. 2005.) As the clump is turbulent and contains many Jeans masses at the onset of dynamical collapse, it quickly fragments and produces a number of relatively massive "cores". These cores, which are denser than the clump material surrounding them, are collapsing on significantly shorter timescales than the entire protocluster clump. Because of the high turbulent/dynamic ambient pressure, the cores are characterized by large densities, reminiscent of the MT03 model, and form individual protostars with high mass infall rates. Although most of these cores have masses exceeding the local Jeans mass, we speculate that further fragmentation will be largely inhibited as the system is already dominated by strongly converging collapse motions as opposed to random turbulent motions ${ }^{2}$. This contrasts with the collapse of massive turbulent cores initially in hydrostatic

\footnotetext{
${ }^{2}$ Strictly speaking, the observations discussed here mostly constrain the motions of the parent clump on scales larger than $\sim 10^{4} \mathrm{AU}$. Higher-resolution observations with the IRAM Plateau de Bure interferometer were undertaken to probe the dynamics and degree of fragmentation of individual MMSs or "cores" on smaller scales and will be reported in a future paper.
} 
equilibrium, which in turn yields multiple low-mass fragments according to recent numerical simulations (Dobbs et al. 2005). In other words, we speculate that the state of global, dynamical collapse of the parent protocluster clump will strongly limit the process of sub-fragmentation in the cores, which may help to solve one of the main problems of the MT03 model of massive star formation. In our radiative transfer model of NGC 2264-C, the 3D turbulent velocity dispersion $\sigma_{\text {turb-3D }}=0.81 \mathrm{~km} \mathrm{~s}^{-1}$ is smaller than the infall velocity $V_{\text {inf }}=1.3 \mathrm{~km} \mathrm{~s}^{-1}$ at $r \lesssim$ $3.5 \times 10^{4} \mathrm{AU}$, so that coherent infall motions dominate turbulent motions at small radii. While the estimated level of turbulence is probably still sufficient to alter the gas motions from a pure radial infall pattern, we thus do not expect turbulent Jeans-type fragmentation to dominate in the inner $\$ 3.5 \times 10^{4}$ AU region. On the contrary, our observational results rather suggest that two or more cores are in the process of merging in this central region.

We conclude that the millimeter continuum sources identified in the central part of NGC 2264-C are the probable precursors of intermediate-/high-mass stars. Powerful winds are then likely to be generated that can clear away most of the protocluster gas and thus effectively stop the global collapse of the system. In this way, the protocluster clump NGC 2264-C will probably evolve into a revealed star cluster similar to the neighboring near-IR cluster discussed by Lada \& Lada (2003) (see Fig. 16).

\section{Summary}

The main results of our comprehensive millimeter study of the cluster-forming clumps NGC 2264-C and NGC 2264-D are as follows:

1. From our dust continuum maps, we extracted a total of 27 compact millimeter sources (MMSs) in NGC 2264, 12 in clump C, and 15 in clump D. These MMSs have a typical diameter $\sim 0.04 \mathrm{pc}$ and masses ranging from $\sim 2$ to $41 M_{\odot}$. The median mass of the MMSs is slightly larger in NGC $2264-\mathrm{C}\left(\sim 10 M_{\odot}\right.$ versus $\sim 6 M_{\odot}$ in NGC $\left.2264-\mathrm{D}\right)$, which is the more massive clump with a total gas mass $\sim 1650 M_{\odot}$ (compared to $\sim 1310 M_{\odot}$ for NGC 2264-D). In both cases, the mass contained in the MMSs amounts to less than $10 \%$ of the total mass of the parent clump.

2. The MMSs of NGC 2264 exhibit broad $\mathrm{N}_{2} \mathrm{H}^{+}(1-0)$ linewidths tracing supersonic line-of-sight velocity dispersions $\sigma_{\text {line }} \sim 0.7 \mathrm{~km} \mathrm{~s}^{-1}$ ) on $\sim 0.1 \mathrm{pc}$ scales. This is $\sim$ twice and $\sim$ five times as large as the line-of-sight velocity dispersions measured on similar scales toward the dense cores of the $\rho$-Ophiuchi main cloud and the isolated prestellar cores of Taurus, respectively, suggesting that the MMSs of NGC 2264 are significantly more turbulent than the dense cores of both $\rho$-Oph and Taurus.

3. As much as $70 \%$ of the MMSs of NGC 2264-C are not starless but already contain candidate Class 0 protostellar objects traced by the presence of shocked $\mathrm{H}_{2}$ jets. In NGC 2264-D, we have direct evidence of the protostellar nature of only $25 \%$ of the MMSs from the close association with 2MASS and/or MSX near-/mid-IR source(s). This apparent difference between NGC 2264-C and NGC 2264-D may be an artifact due to the lack of systematic jet/outflow surveys in NGC 2264-D. The high percentage of candidate Class 0 objects in NGC 2264-C may result from a short, efficient episode of star formation, as expected in the case of triggered cloud collapse.

4. In NGC 2264-C, we observe widespread blue-skewed "infall" line profiles in optically thick tracers such as $\mathrm{HCO}^{+}(3-2)$ or $\mathrm{CS}(3-2)$, consistent with the presence of large-scale infall motions in this clump as previously suggested by Williams \& Garland (2002).

5. Furthermore, our mapping in low optical depth tracers such as $\mathrm{N}_{2} \mathrm{H}^{+}(1-0)$ or $\mathrm{H}^{13} \mathrm{CO}^{+}(1-0)$ reveals a new velocity feature in the central part of NGC 2264-C, in the form of a sharp velocity discontinuity $\sim 2 \mathrm{~km} \mathrm{~s}^{-1}$ in amplitude centered on the millimeter source C-MM3.

6. Taken together, we interpret these two velocity features as the signatures of the large-scale collapse of a cylinder-like, filamentary structure along its long axis. Radiative transfer calculations confirm this view and suggest an infall velocity $V_{\text {inf }}=1.3 \mathrm{~km} \mathrm{~s}^{-1}$ along the main axis of the NGC 2264-C prolate clump, corresponding to a total inflow rate $\sim 3 \times$ $10^{-3} M_{\odot} \mathrm{yr}^{-1}$ toward the central Class 0 object C-MM3.

7. We also found evidence of both infall and rotation motions toward the Class 0 object D-MM1 in the NGC 2264-D clump. Based on radiative transfer modelling of our $\mathrm{HCO}^{+}$ and CS observations, we estimate an infalling velocity $V_{\text {inf }}=0.1 \mathrm{~km} \mathrm{~s}^{-1}$ which, given the high density of D-MM1, corresponds to a mass infall rate $\sim 1.1 \times 10^{-4} M_{\odot} \mathrm{yr}^{-1}$. Such an infall rate should be high enough to overcome the radiation pressure of a massive $\gtrsim 10 M_{\odot}$ star.

8. Comparison of our observations with existing scenarios for massive star formation suggests that we are witnessing the initial stages of the formation of a high-mass $\sim 10-20 M_{\odot}$ star in the central part of NGC 2264-C. Based on this comparison, we propose a picture of high-mass star formation in protoclusters that is intermediate between the highly dynamic scenario of Bonnell et al. (1998) and the turbulent core scenario of McKee \& Tan (2003). In this observationally-driven picture, the large-scale, dynamical collapse of a massive, initially unstable clump would lead to the formation of a turbulent, massive, and ultra-dense core with properties reminiscent of the McKee \& Tan model, through the gravitational merger of two or more intermediate-mass Class 0 protostellar cores in the central part of the clump, while the potential of the system is still gas-dominated.

Acknowledgements. We would like to thank Frédérique Motte for providing her source extraction procedure and for useful discussions on protoclusters. We also thank Didier Despois and Stephan Blinder for letting us use their radiative transfer code, MAPYSO. We acknowledge stimulating discussions with Patrick Hennebelle on cloud fragmentation and collapse. We are grateful to the anonymous referee for constructive comments, which helped us improve the clarity of the paper. The observations presented in this paper were carried out with the IRAM $30 \mathrm{~m}$ telescope; IRAM is supported by INSU/CNRS (France), MPG (Germany), and IGN (Spain). 


\section{References}

Adams, F., \& Myers, P. 2001, ApJ, 553, 744

Allen, D. 1972, ApJ, 172, 55

André, P., Ward-Thompson, D., \& Barsony, M. 1993, ApJ, 406, 122 André, P. 2002, in The Evolution of Galaxies, ed. M. Sauvage, G. Stansińska, \& D. Schaerer, Ap\&SS, 281, 51

André, P., Bouwman, J., Belloche, A., \& Hennebelle, P. 2004, in Magnetic Field and Star Formation: Theory Versus Observations, ed. A. Gómez De Castro et al., Ap\&SS, 292, 325

Bacmann, A., Lefloch, B., Ceccarelli, C., et al. 2002, A\&A, 389, 6

Bate, M., Bonnell, I., \& Bromm, V. 2003, MNRAS, 339, 577

Belloche, A., André, P., \& Motte, F. 2001, in From Darkness to Light: Origin and Evolution of Young Stellar Clusters, ed. T. Montmerle, $\&$ Ph. André, ASP Conf. Ser., 243 (San Francisco: ASP), 313

Belloche, A., André, P., Despois, D., \& Blinder, S. 2002, A\&A, 393, 927

Belloche, A., \& André, P. 2004, A\&A, 419, 35

Bergin, E., \& Langer, W. 1997, ApJ, 486, 316

Bergin, E., Unggerechts, H., Goldsmith, P., et al. 1997, ApJ, 482, 267

Bernes, C. 1978, Stockholm Observatory Report No. 15 + Erratum

Bernes, C. 1979, A\&A, 73, 67

Bertoldi, F., \& McKee, C. 1992, ApJ, 395, 140

Blinder, S. 1997, Thèse, Université de Bordeaux I

Bonnell, I., Bate, M., \& Zinnecker, H. 1998, MNRAS, 298, 93

Bonnell, I., Bate, M., Clarke, C., \& Pringle, J. 2001, MNRAS, 323, 785

Bonnell, I., \& Bate, M. 2002, MNRAS, 336, 705

Broguière, D., Neri, R., \& Sievers, A. 1995, NIC bolometer users guide, in IRAM internal report

Caselli, P., Benson, P., Myers, P., \& Tafalla, M. 2002, ApJ, 572, 238

Clarke, C., Bonnell, I., \& Hillenbrand, L. 2000, in Protostars and Planets IV, ed. V. Mannings, A. Boss, \& S. Russell (Tucson: Univ. Arizona Press), 151

Di Francesco, J., Myers, P., Wilner, D., Ohashi, N., \& Mardones, D. 2001, ApJ, 562, 770

Dobbs, C., Bonnell, I., \& Clarke, P. 2005, MNRAS, 360, 2

Encrenaz, P., Falgarone, E., \& Lucas, R. 1975, A\&A, 44, 73

Evans, N. 1999, ARAA, 37, 311

Genzel, R., Downes, D. 1977, A\&AS, 30, 145

Greene, T., Wilking, B., André, P., Young, E., \& Lada, C. 1994, ApJ, 434,614

Haschick, A., Menten, K., \& Baan, W. 1990, ApJ, 354, 556

Hennebelle, P., Whitworth, A., Gladwin, P., \& André, P. 2003, MNRAS, 340, 870

Jijina, J., \& Adams, F. 1996, ApJ, 462, 874

Jijina, J., Myers, P. C., \& Adams, F. 1999, ApJS, 125, 161

Klessen, R., \& Burkert, A. 2000, ApJS, 128, 287

Krügel, E., Guesten, R., Schulz, A., \& Thum, C. 1987, A\&A, 185, 283

Lada, C., Young, E., \& Greene, T. 1993, ApJ, 408, 471
Lada, C., \& Lada, E. 2003, ARA\&A, 41, 57

Loren, R., Wooten, A., \& Wilking, B. 1990, ApJ, 365, 269

Margulis, M., Lada, C., \& Snell, R. 1988, ApJ, 333, 316

Margulis, M., Lada, C., \& Young, E. 1989, ApJ, 345, 906

Matzner, C., \& McKee, C. 2000, ApJ, 545, 364

Mc Kee, C., \& Tan, J. 2002, Nature, 416, 59

Mc Kee, C., \& Tan, J. 2003, ApJ, 585, 850 (MT03)

Mendoza, E., Rodriguez, L., Chavarria-K, C., \& Neri, L. 1990, MNRAS, 246, 518

Minier, V., Conway, J., \& Booth, R. 2001, A\&A, 369, 278

Motte, F., André, P., \& Neri 1998, A\&A, 336, 150 (MAN98)

Motte, F., \& André, P. 2001, A\&A, 365, 440

Motte, F., Schilke, P., \& Lis, D. 2003, ApJ, 582, 277

Motte, F., Bontemps, S., Schilke, P., et al. 2005, in Massive star birth: a crossroads of astrophysics, IAU Symp., 227, ed. R. Cesaroni, E. Churchwell, M. Felli, \& M. Walmsley

Myers, P. 1998, ApJ, 496, 109

Nakano, M., Sugitani, K., \& Morita, K. 2003, PASJ, 55, 321

Ohashi, N. 1999, in Star Formation 1999, ed. T. Nakamoto, Nobeyama Radio Observatory, 129

Padoan, P., \& Nordlund, A. 2002, ApJ, 576, 870

Schreyer, K., Helmich, F., Van Dishoeck, E., \& Henning, T. 1997, A\&A, 326, 347

Schreyer, K., Stecklum, B., Linz, A., \& Henning, T. 2003, ApJ, 599, 335

Shu, F., Adams, F., \& Lizano, S. 1987, ARA\&A, 25, 23

Shu, F., Li, Z.-Y., \& Allen, A. 2004, ApJ, 601, 930

Stahler, S., Palla, F., \& Ho, P. 2000, in Protostars and Planets IV, ed. V. Mannings, A. Boss, \& S. Russell (Tucson: Univ. Arizona Press), 327

Starck, J.-L., Bijaoui, A., \& Murtagh, F. 1995, in CVIP: Graphical Models and Image Processing, 57, 5, 420

Stutzki, J., \& Güsten, R. 1990, ApJ, 356, 513

Tafalla, M., et al. 2002, ApJ, 569, 81

Testi, L., Palla, F., \& Natta, A. 1999, A\&A, 342, 823

Thompson, R., Corbin, M., Young, E., \& Schneider, G. 1998, ApJ, 492, 177

Wang, H., Yang, J., Wang, M., \& Yan, J. 2002, A\&A, 389, 1015

Ward-Thompson, D., Motte, F., \& André, P. 1999, MNRAS, 305, 143

Ward-Thompson, D., Zylka, R., Mezger, P., \& Sievers, A. 2000, A\&A, 355,1122

Wilking, B., \& Lada, C. 1983, ApJ, 274, 698

Williams, J., \& Garland, C. 2002, ApJ, 568, 259

Wolf-Chase, G., Walker, C., \& Lada, C. 1995, ApJ, 442, 197

Wolf-Chase, G., Moriarty-Schieven, G., Fich, M., \& Barsony, M. 2003, MNRAS, 344, 809

Wolfire, M., \& Cassinelli, J. 1987, ApJ, 319, 850

Zinnecker, H., McCaughrean, M., Rayner, J., Wilking, B., \& Moneti, A. 1993, in Protostars and Planets III, ed. E. Levy, \& J. Lunine (Tucson: Univ. Arizona Press), 429 